\title{
The inverse demand oxa-Diels-Alder reaction of resorcinarenes: experimental and theoretical analysis of regioselectivity and diastereoselectivity
}

\author{
K. Stefańska, H. Jędrzejewska, M. Wierzbicki, A. Szumna, * W. Iwanek*
}

ELECTRONIC SUPLEMENTARY INFORMATION

\section{Table of contents}

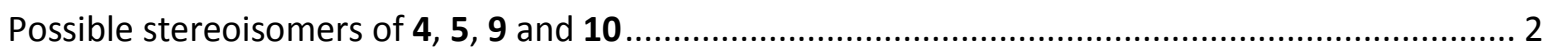

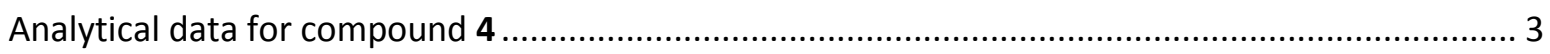

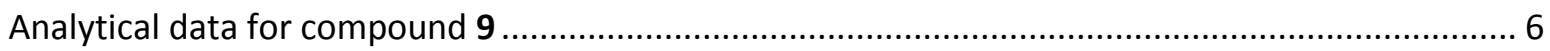

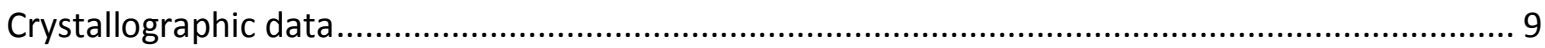

Chromatographic resolution of rac-4 and ECD spectra of enantiomers ......................................... 11

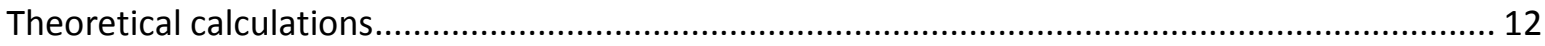

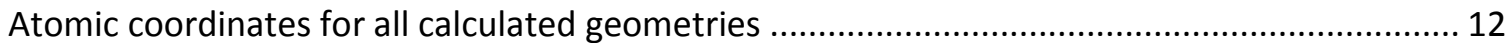




\section{Possible stereoisomers of 4, 5, 9 and 10}
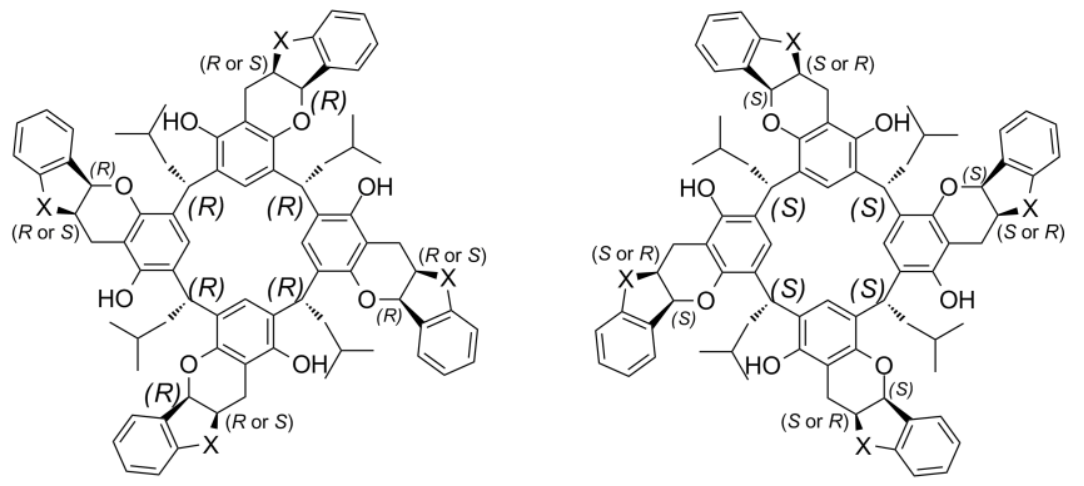

$\begin{array}{ll}\text { (all- } R, R, \text { all- } R \text { )-4: } & \mathrm{X}=\mathrm{O} \\ \text { (all-S, } R, \text { all- } R \text { )-9: } & \mathrm{X}=\mathrm{CH}_{2}\end{array}$

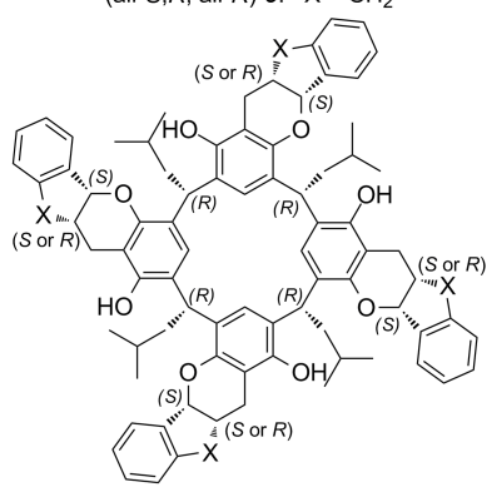

(all-S,S, all-S)-4: $X=0$

(all-R,S, all-S)-9: $\mathrm{X}=\mathrm{CH}_{2}$

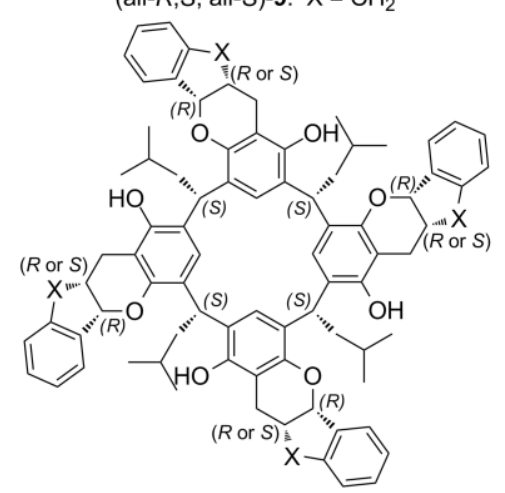

(all-S,S, all-R)-4: $\mathrm{X}=\mathrm{O}$ (all-R,S, all-R)-9: $\mathrm{X}=\mathrm{CH}_{2}$

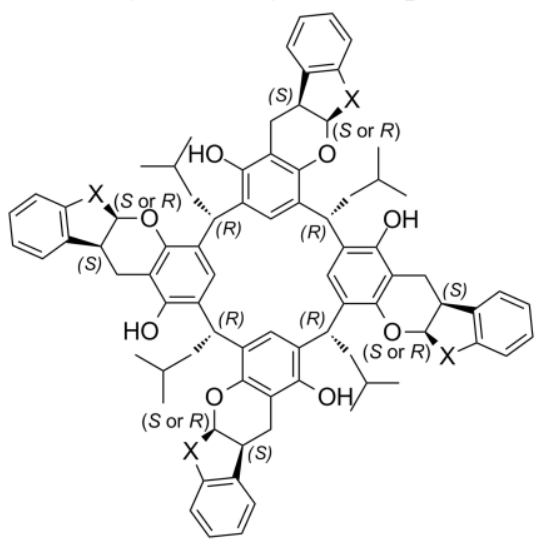

(all-S, $S$, all-R)-5: $\mathrm{X}=\mathrm{O}$

(all- $R, S$, all-R)-10: $\mathrm{X}=\mathrm{CH}_{2}$

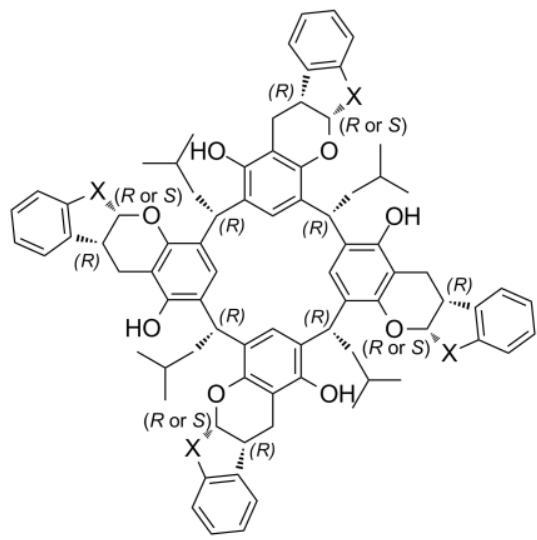

(all- $R, R$, all- $R$ )-5: $\quad \mathrm{X}=0$

(all-S, $R$, all- $R$ )-10: $\mathrm{X}=\mathrm{CH}_{2}$

(all-R,R, all-S)-4: $\mathrm{X}=\mathrm{O}$

(all-S,R, all-S)-9: $\mathrm{X}=\mathrm{CH}_{2}$

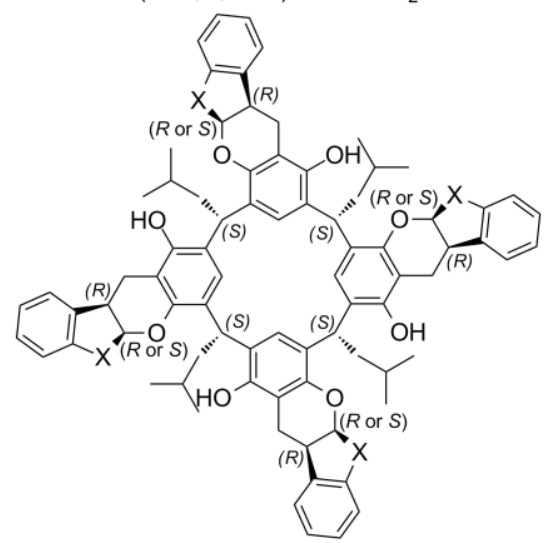

(all- $R, R$, all-S)-5: $\quad \mathrm{X}=\mathrm{O}$

(all-R,R, all-S)-5: $\mathrm{X}=\mathrm{O}$
(all-S, $R$, all-S)-10: $\mathrm{X}=\mathrm{CH}_{2}$

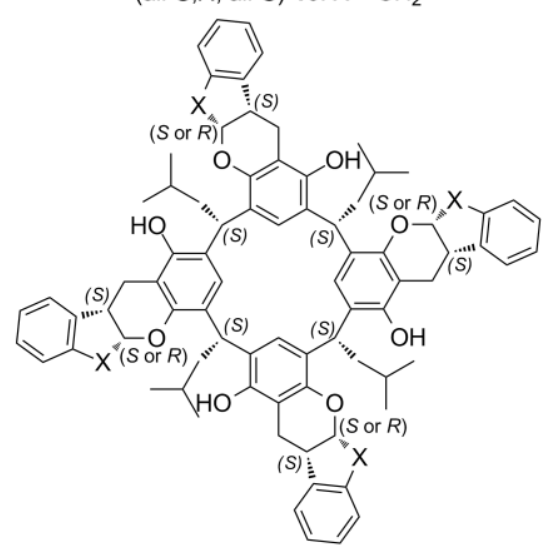

(all-S,S, all-S)-5: $\mathrm{X}=0$

(all-R,S, all-S)-10: $\mathrm{X}=\mathrm{CH}_{2}$

Figure S1. All possible enantiomers of 4, 5, 9 and 10. 


\section{Analytical data for compound 4}

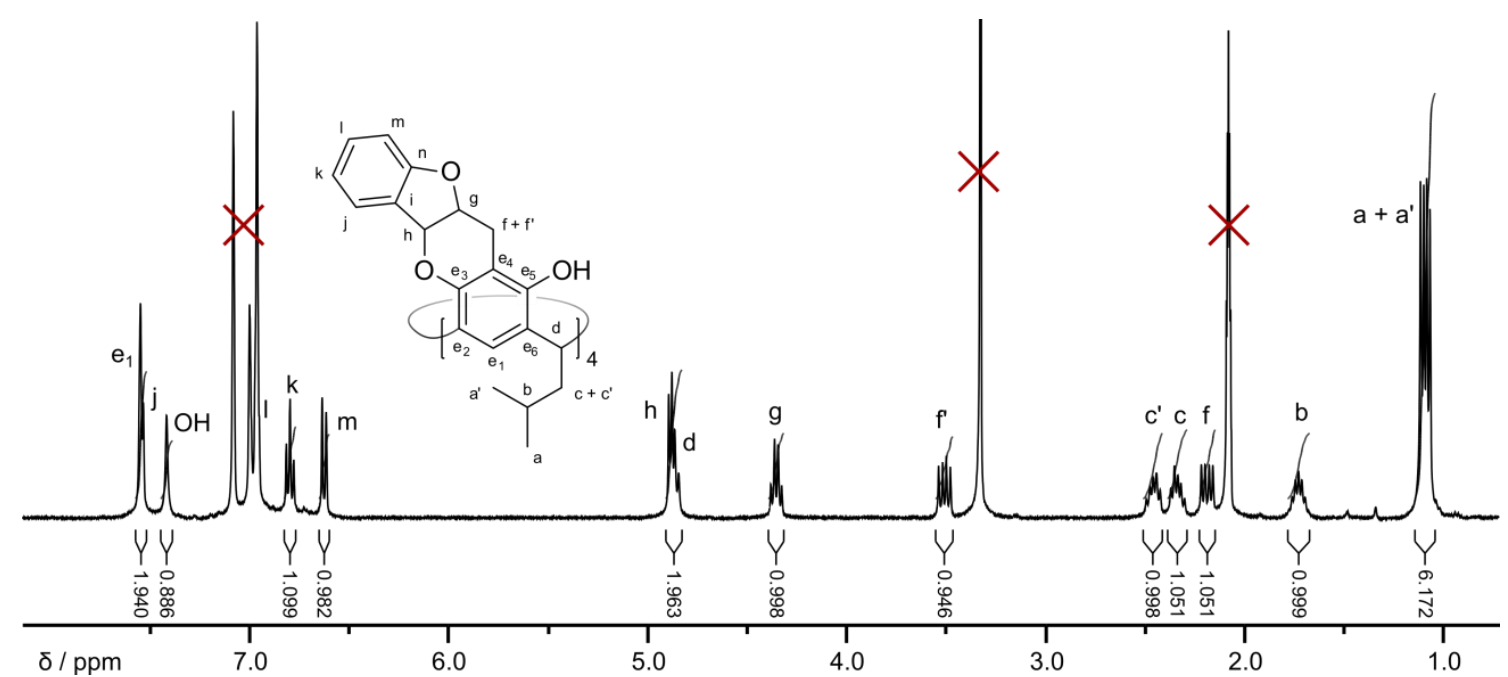

Figure S2. ${ }^{1} \mathrm{H}$ NMR spectrum of $4\left(400 \mathrm{MHz}\right.$, toluene- $\left.\mathrm{d}_{8}\right)$. The / proton signal is overlapping with the residual signals of deuterated toluene and cannot be integrated. The signals of 1,4-dioxane and toluene- $d_{8}$ have been crossed out.
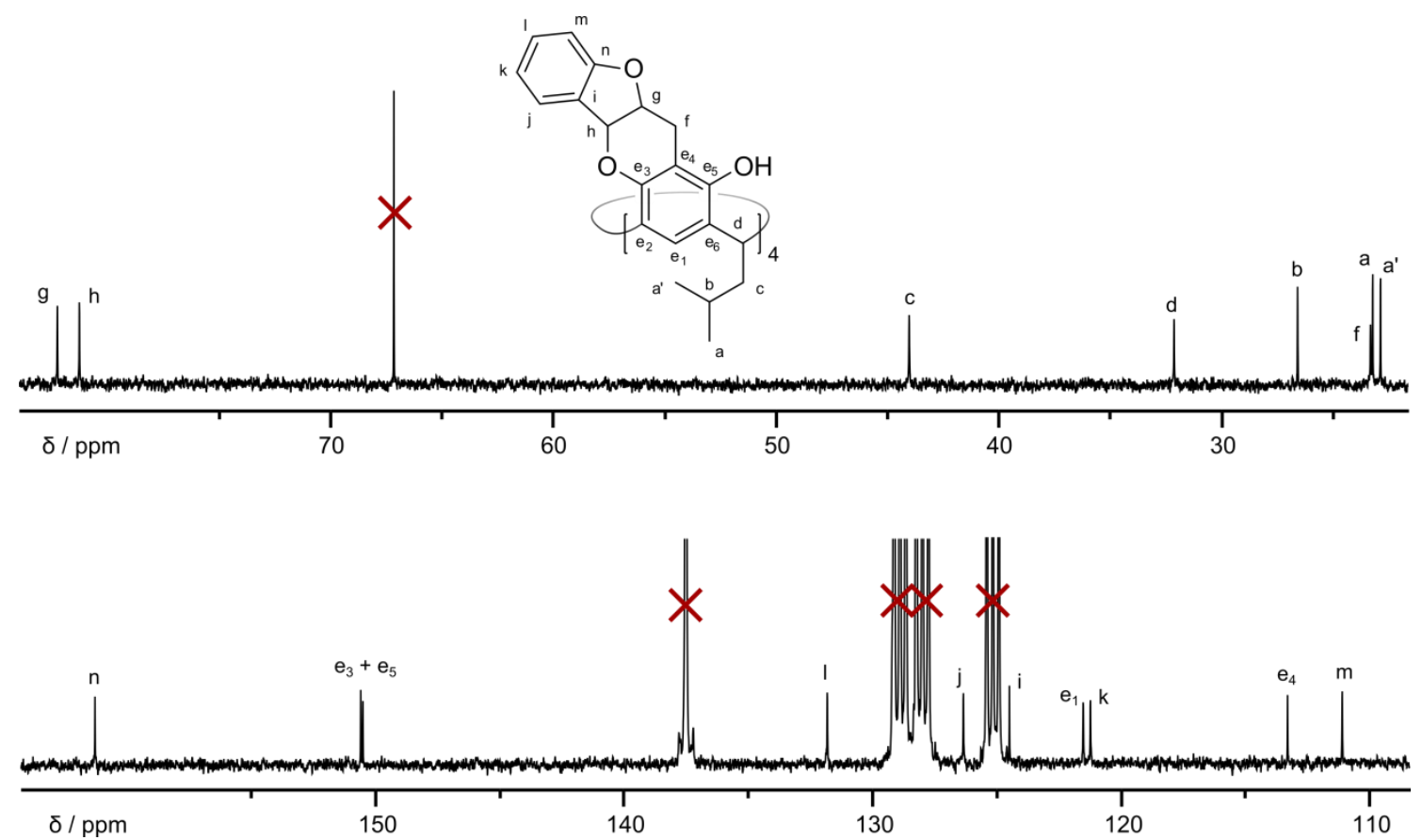

Figure S3. ${ }^{13} \mathrm{C}$ NMR spectrum (relevant regions) of 4 (100 MHz, toluene- $\left.\mathrm{d}_{8}\right)$. The $\mathrm{e}_{2}$ and $\mathrm{e}_{6}$ carbon signals are overlapping with the residual signals of deuterated toluene, as can be deduced from 2D NMR data. The signals of 1,4-dioxane and toluene- $d_{8}$ have been crossed out. 


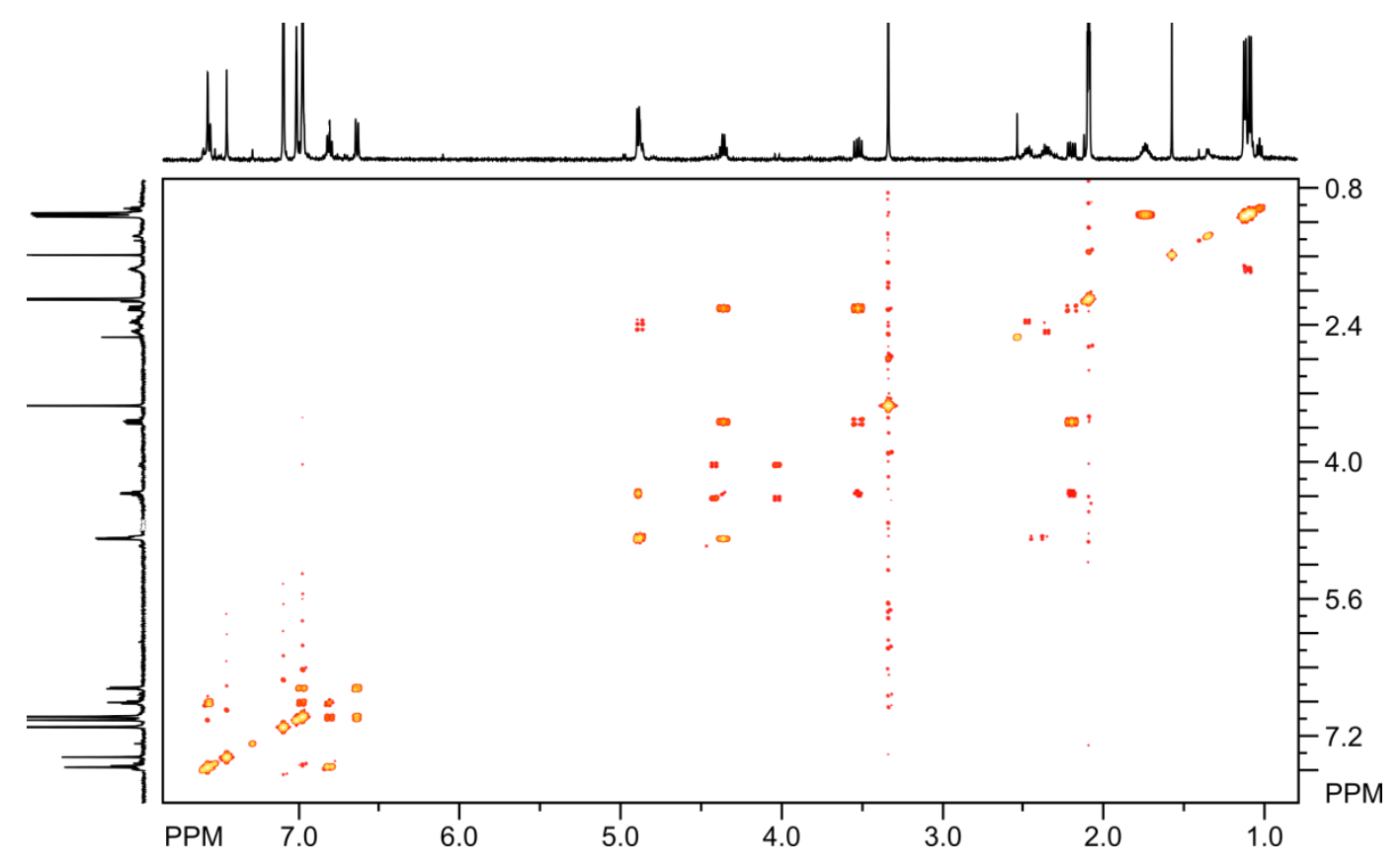

Figure 4. COSY spectrum of $4\left(500 \mathrm{MHz}\right.$, toluene- $\left.\mathrm{d}_{8}\right)$.
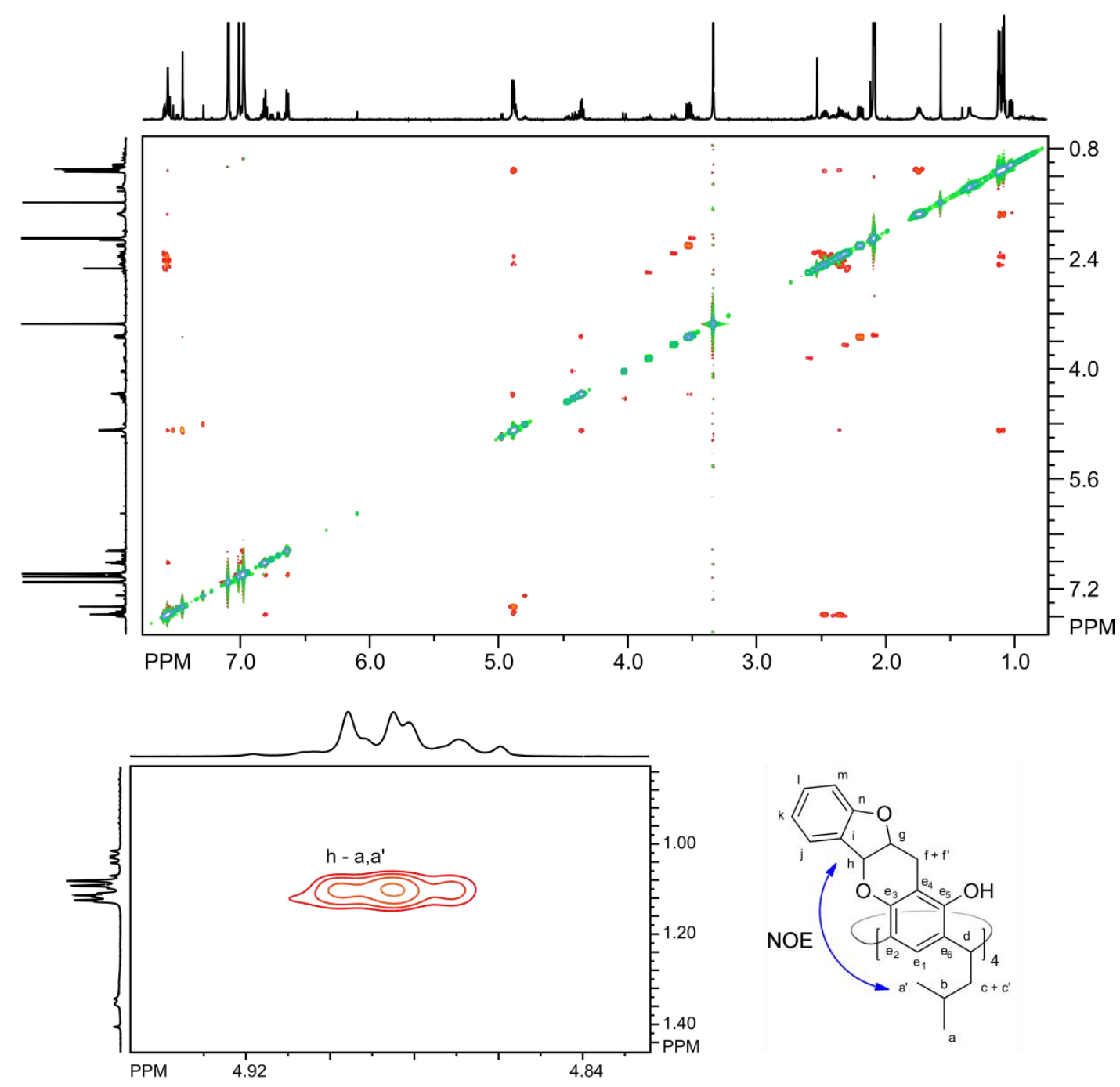

Figure S5. ROESY spectrum of 4 (600 MHz, toluene- $\left.\mathrm{d}_{8}\right)$ : full spectrum (top), most indicative signal (bottom). 


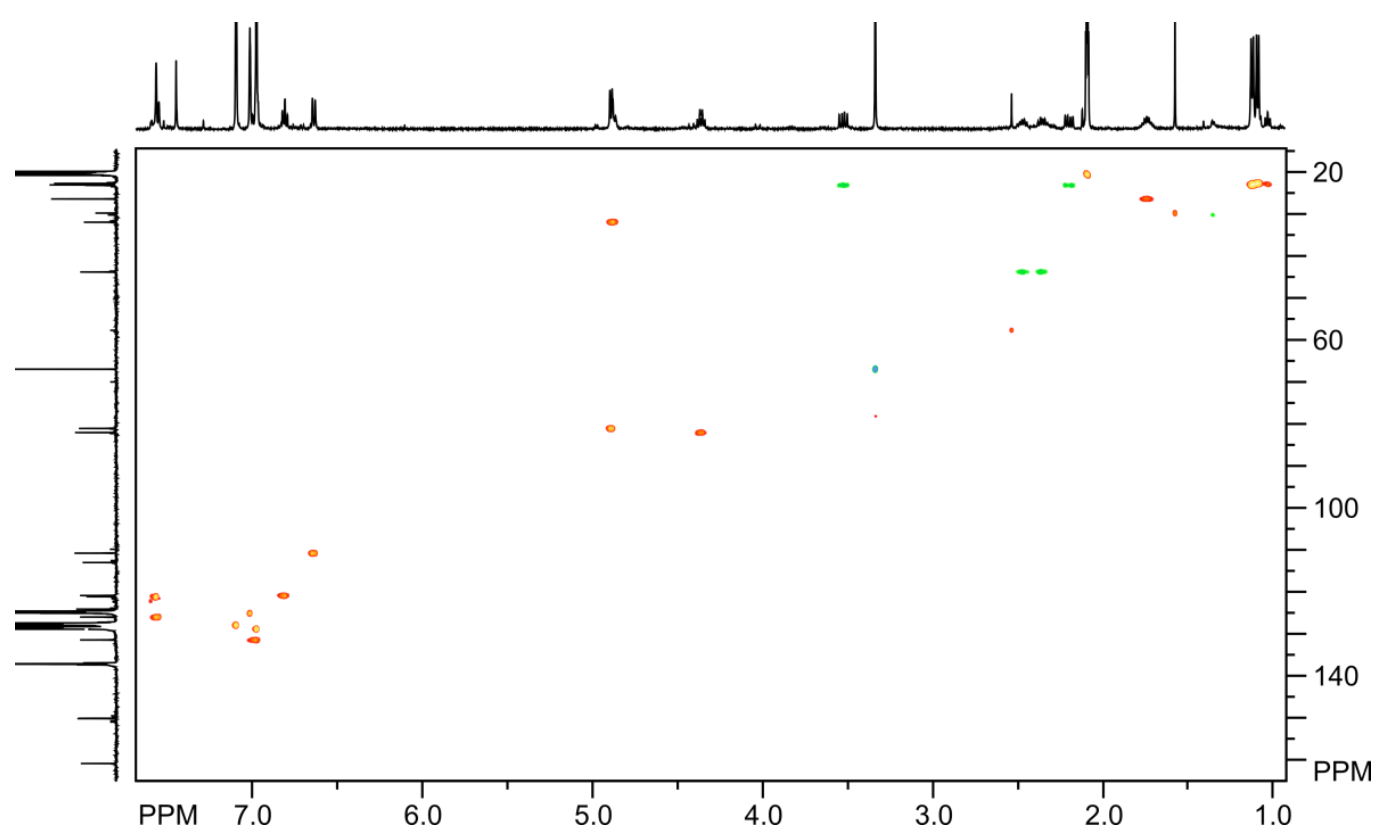

Figure 6. HSQC spectrum of $4\left(500 \mathrm{MHz}\right.$, toluene- $\left.\mathrm{d}_{8}\right)$.

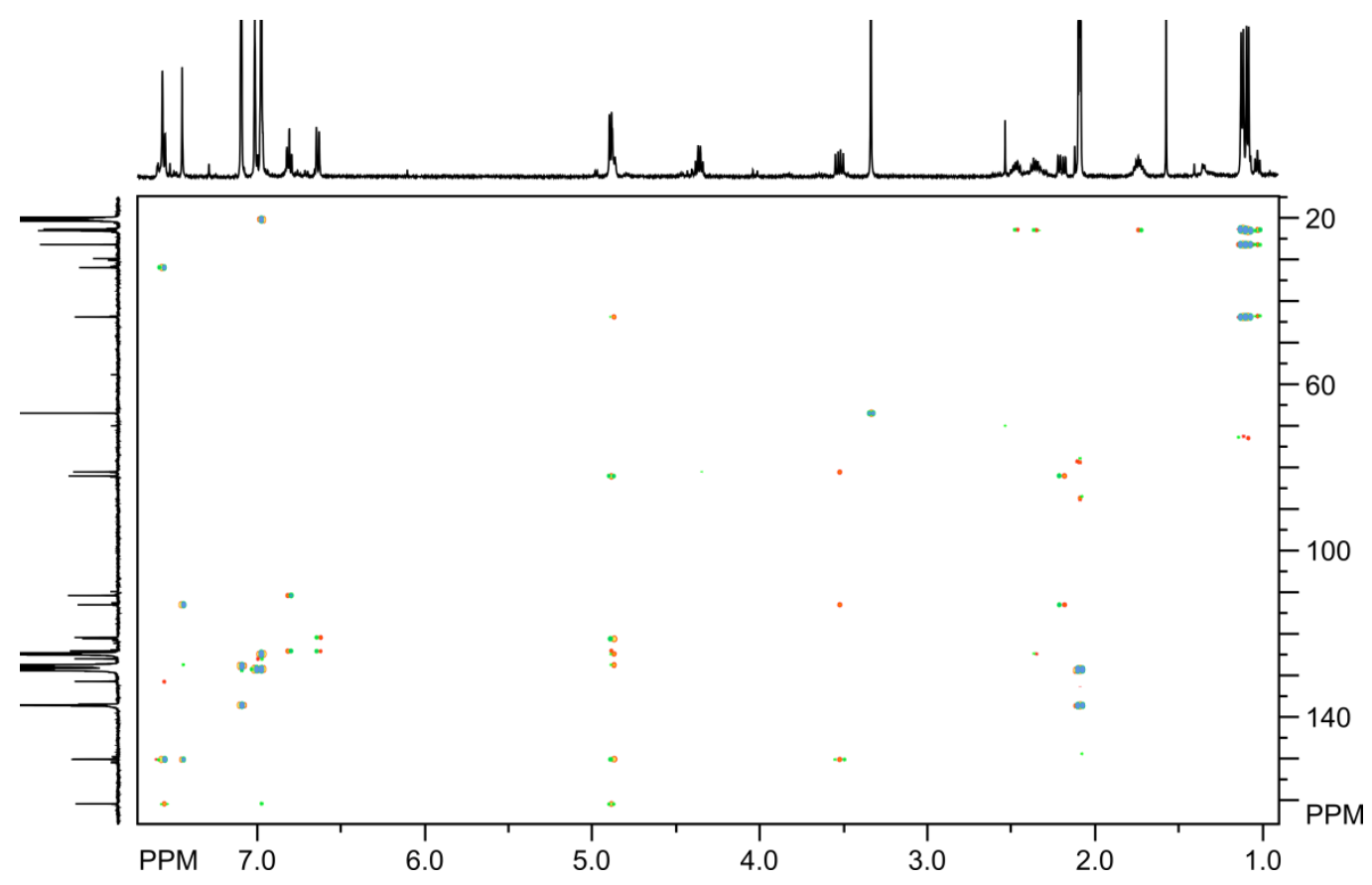

Figure S7. HMBC spectrum of $4\left(500 \mathrm{MHz}\right.$, toluene- $\left.\mathrm{d}_{8}\right)$. 


\section{Analytical data for compound 9}

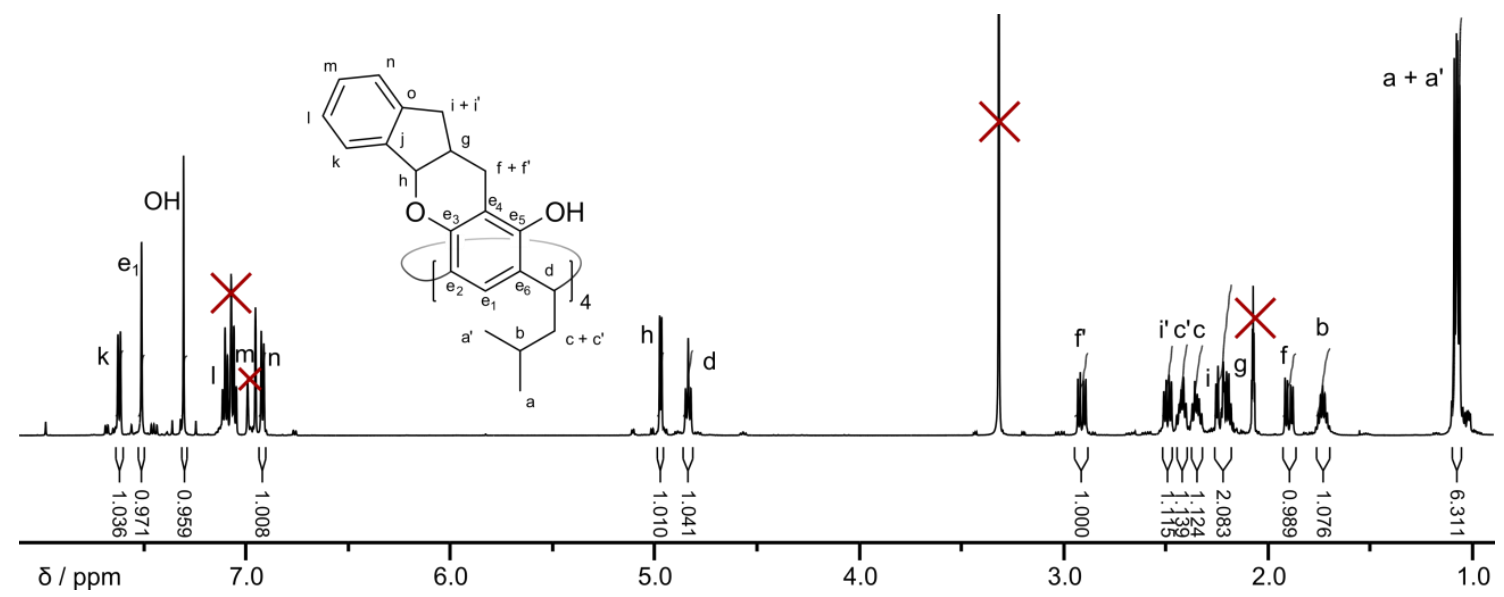

Figure S8. ${ }^{1} \mathrm{H}$ NMR spectrum of $9\left(600 \mathrm{MHz}\right.$, toluene- $\left.\mathrm{d}_{8}\right)$. The / and $m$ proton signals are overlapping with the residual signals of deuterated toluene and cannot be integrated. The signals of 1,4-dioxane and toluene- $d_{8}$ have been crossed out.

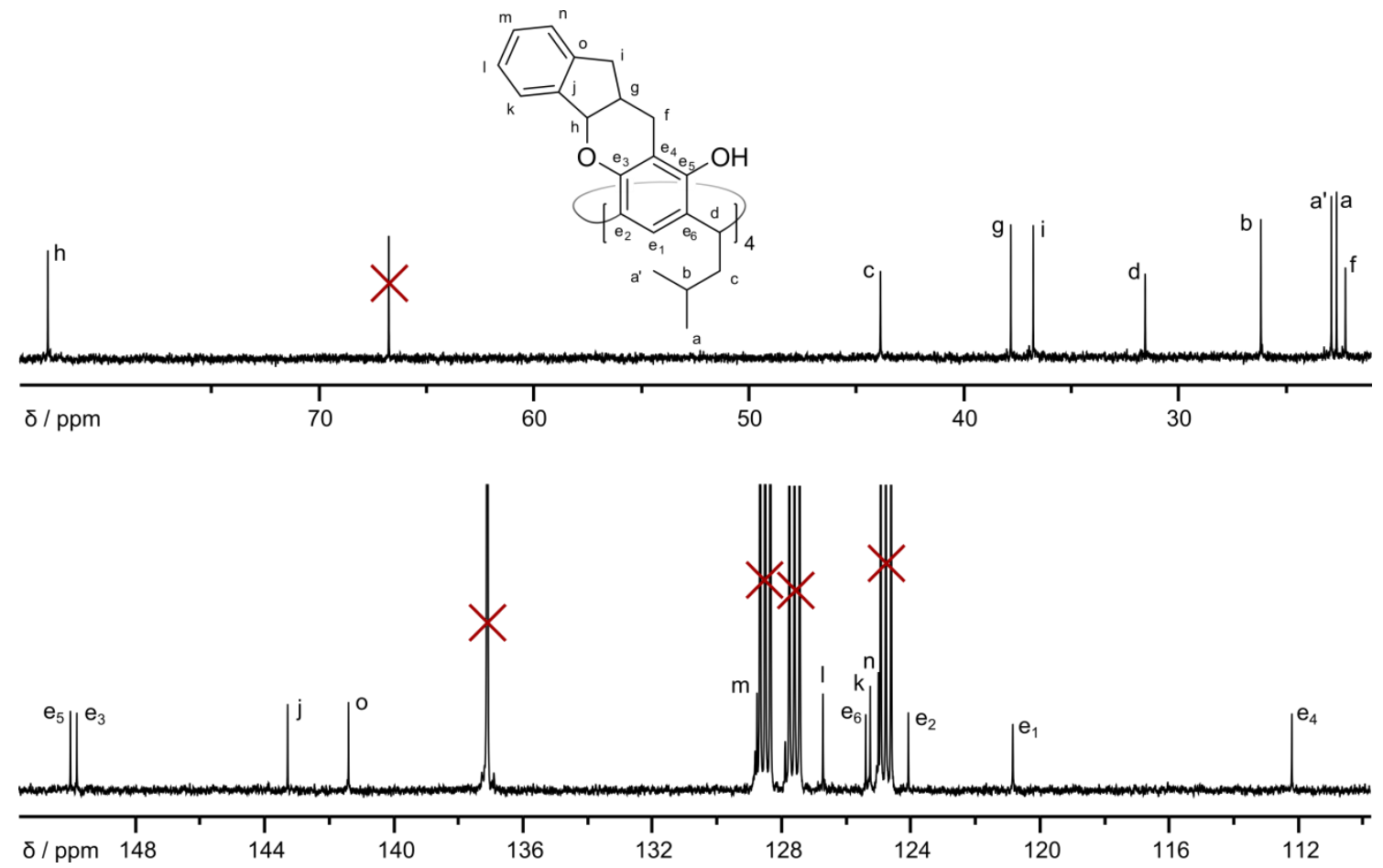

Figure S9. ${ }^{13} \mathrm{C}$ NMR spectrum (relevant regions) of $9\left(150 \mathrm{MHz}\right.$, toluene- $\left.d_{8}\right)$. The signals of 1,4-dioxane and toluene- $d_{8}$ have been crossed out. 

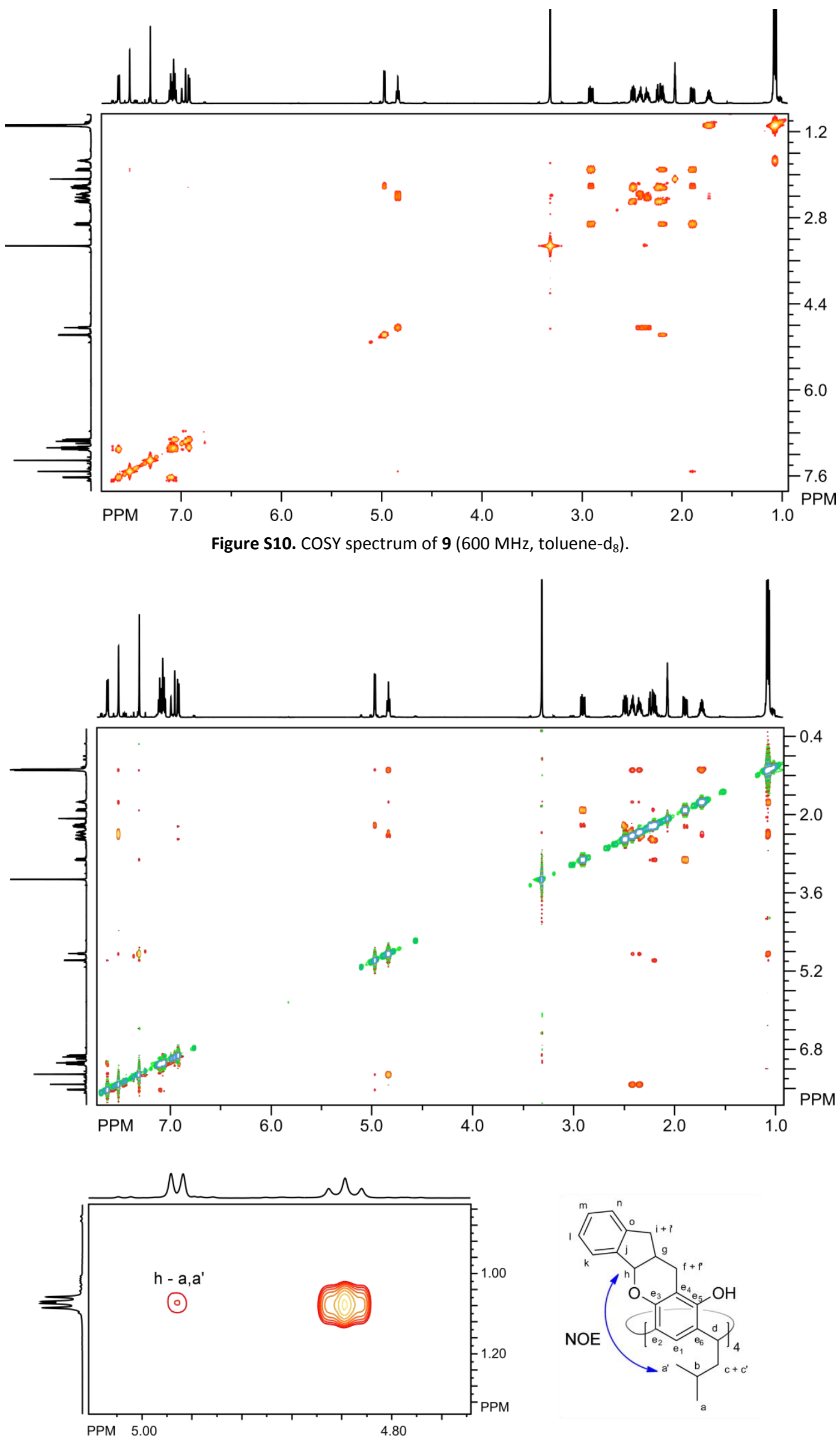

Figure S11. ROESY spectrum of 9 (600 MHz, toluene- $\left.\mathrm{d}_{8}\right)$ : full spectrum (top), most indicative signal (bottom). 


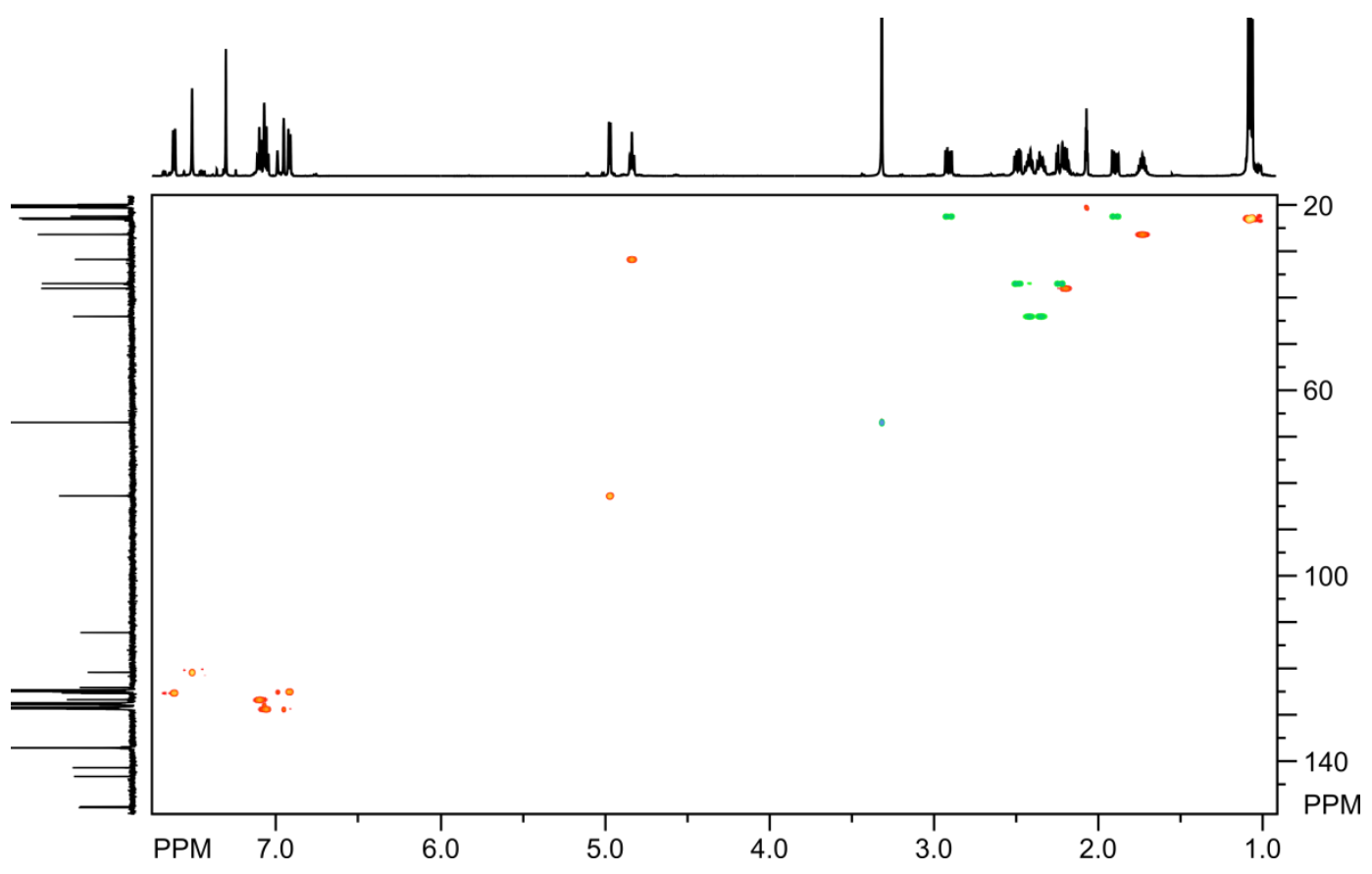

Figure S12. HSQC spectrum of $9\left(600 \mathrm{MHz}\right.$, toluene- $\left.\mathrm{d}_{8}\right)$.

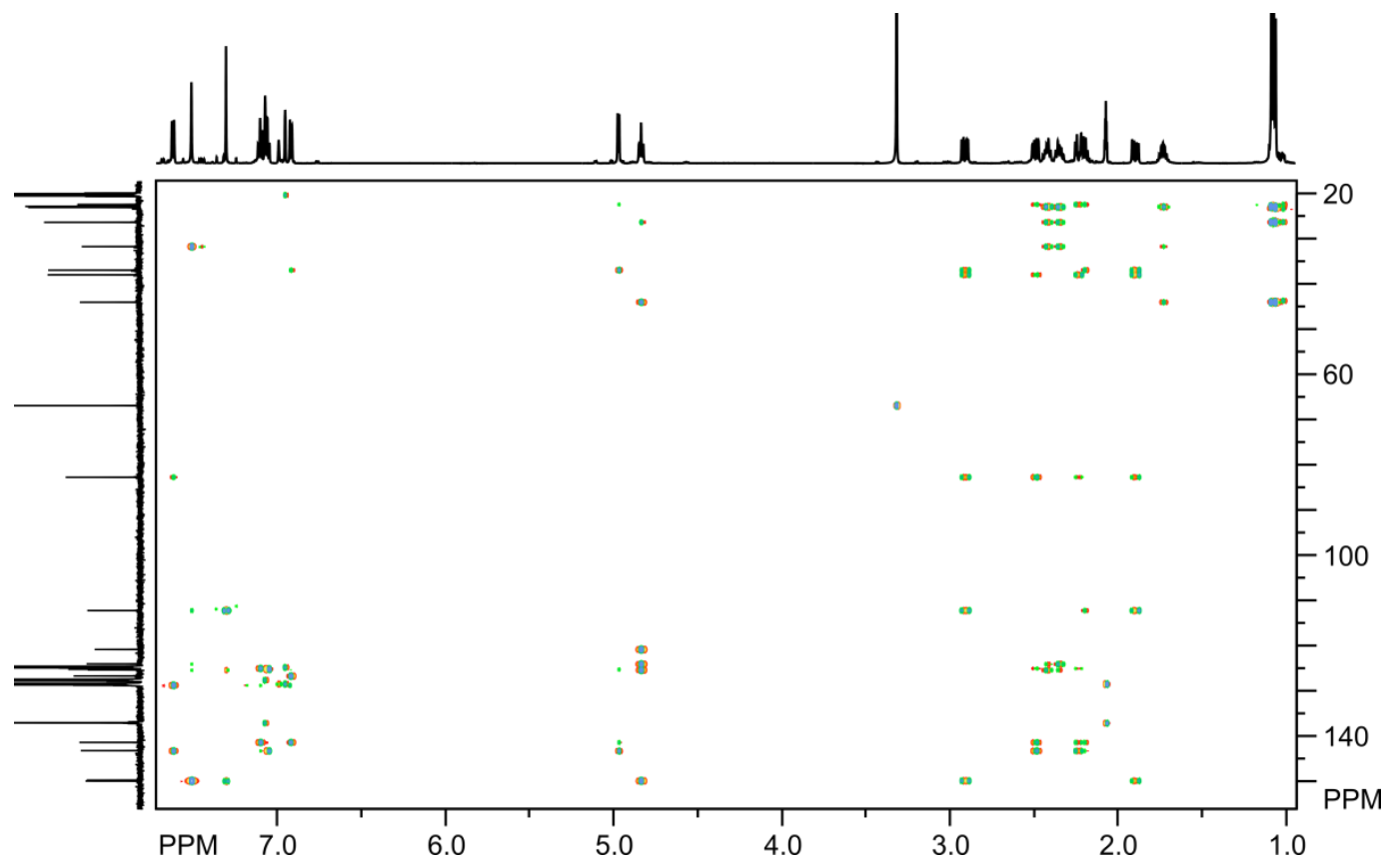

Figure S13. HMBC spectrum of $9\left(600 \mathrm{MHz}\right.$, toluene- $\left.\mathrm{d}_{8}\right)$. 


\section{Crystallographic data}

CCDC 1470016 and 1483833 contain the supplementary crystallographic data for this paper. These data can be obtained free of charge from The Cambridge Crystallographic Data Centre via www.ccdc.cam.ac.uk/data_request/cif.

\begin{tabular}{|c|c|c|}
\hline \multicolumn{3}{|c|}{ Crystal data for cycloaddition products } \\
\hline Compound number & 4 & 9 \\
\hline Moiety formula & $2\left(\mathrm{C}_{20} \mathrm{H}_{20} \mathrm{O}_{6}\right) \times 0.25\left(\mathrm{C}_{4} \mathrm{H}_{8} \mathrm{O}_{2}\right), \times 0.75\left(\mathrm{C}_{7} \mathrm{H}_{8}\right)$ & $\mathrm{C}_{84} \mathrm{H}_{88} \mathrm{O}_{8} \times 2\left(\mathrm{C}_{7} \mathrm{H}_{8}\right)$ \\
\hline Empirical formula & $\mathrm{C}_{46.25} \mathrm{H}_{48} \mathrm{O}_{6.5}$ & $\mathrm{C}_{98} \mathrm{H}_{104} \mathrm{O}_{8}$ \\
\hline Formula weight & 707.87 & 1409.81 \\
\hline Temperature $(\mathrm{K})$ & 100 & 100 \\
\hline Wavelength $(\AA)$ & 1.54184 & 1.54184 \\
\hline Crystal system & tetragonal & triclinic \\
\hline Space group & $\mathrm{P} 4 / \mathrm{n}$ & $\mathrm{P}-1$ \\
\hline $\begin{array}{l}\text { Unit cell dimensions } \\
\mathrm{a} / \mathrm{b} / \mathrm{c}(\AA) \\
\alpha / \beta / \gamma\left({ }^{\circ}\right)\end{array}$ & $\begin{array}{c}19.6918(10) / 19.6918(10) / 19.3835(2) \\
\\
90 / 90 / 90\end{array}$ & $\begin{array}{c}14.2464(4) / 15.6824(6) / 21.1893(6) \\
77.808(3) / 89.936(2) / 68.049(3)\end{array}$ \\
\hline Unit cell volume $\left(\AA^{3}\right)$ & $7516.28(11)$ & $4276.0(3)$ \\
\hline Z & 8 & 2 \\
\hline Calculated density $\left(\mathrm{g} / \mathrm{cm}^{3}\right)$ & 1.251 & 1.095 \\
\hline Absorption coefficient $\left(\mathrm{mm}^{-1}\right)$ & 0.656 & 0.529 \\
\hline $\mathrm{F}(000)$ & 3020 & 1512 \\
\hline$\theta$ range for data collection $\left(^{\circ}\right)$ & $69.902-3.174$ & $70.208-3.357$ \\
\hline Index ranges & $\begin{array}{l}-23<\mathrm{h}<16 \\
-23<\mathrm{k}<22 \\
-23<\mathrm{l}<23\end{array}$ & $\begin{array}{l}-17<\mathrm{h}<15 \\
-18<\mathrm{k}<18 \\
-25<\mathrm{l}<21\end{array}$ \\
\hline Reflections collected & 29630 & 32476 \\
\hline Independent reflections & $7089\left(R_{\text {int }}=0.0229\right)$ & $15903\left(R_{\text {int }}=0.0299\right)$ \\
\hline Completeness to $\theta_{\max }$ & 0.993 & 0.999 \\
\hline \multicolumn{3}{|c|}{ Refinement statistics } \\
\hline Final $R$ indices $[>2 \sigma(I)]$ & 0.0618 & 0.0616 \\
\hline $\mathrm{R}$ indices [all data] & 0.0693 & 0.0707 \\
\hline Goodness-of-fit & 0.871 & 1.049 \\
\hline Largest diff. peak and hole $\left(\mathrm{e} \AA^{-3}\right)$ & $0.814 /-0.75$ & $0.678 /-0.349$ \\
\hline
\end{tabular}

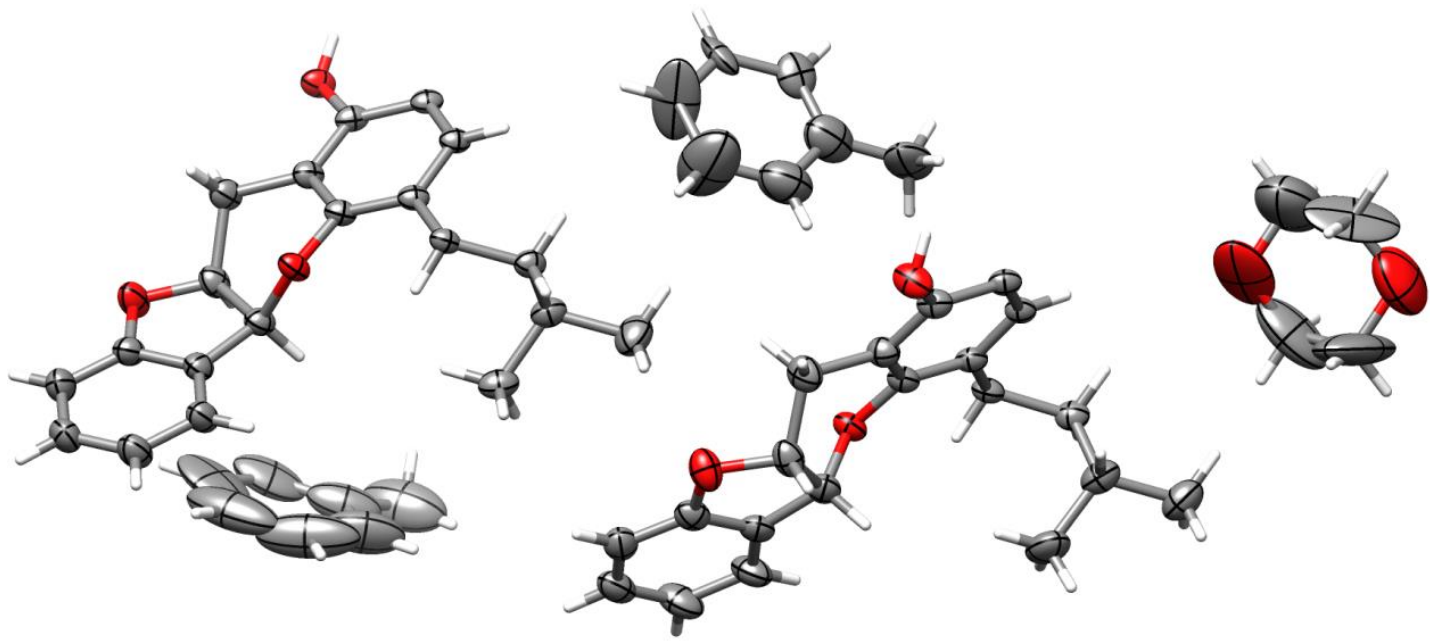

Figure 14. ORTEP representation of crystallographically independent part of 4 . Thermal ellipsoids are depicted at $50 \%$ probability. 


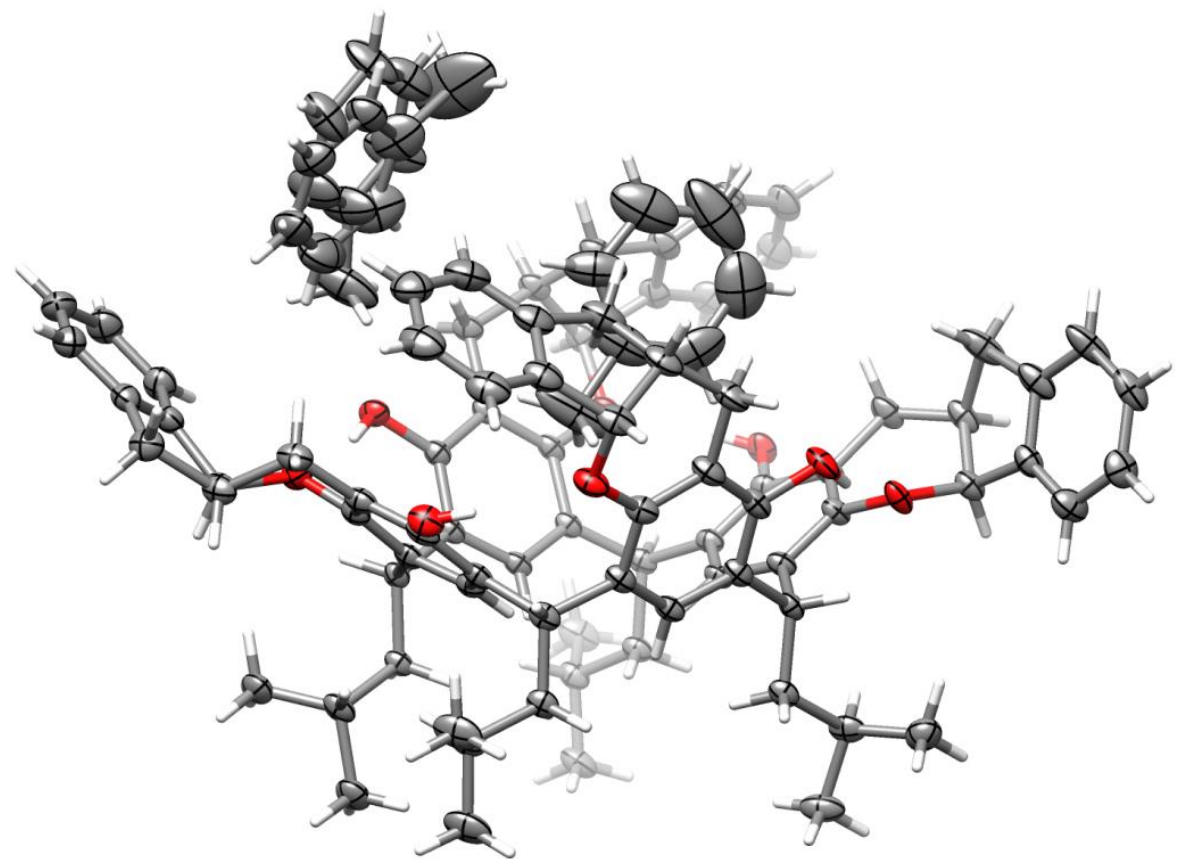

Figure 15. ORTEP representation of crystallographically independent part of $\mathbf{9}$. Thermal ellipsoids are depicted at $50 \%$ probability. 


\section{Chromatographic resolution of rac-4 and ECD spectra of enantiomers}

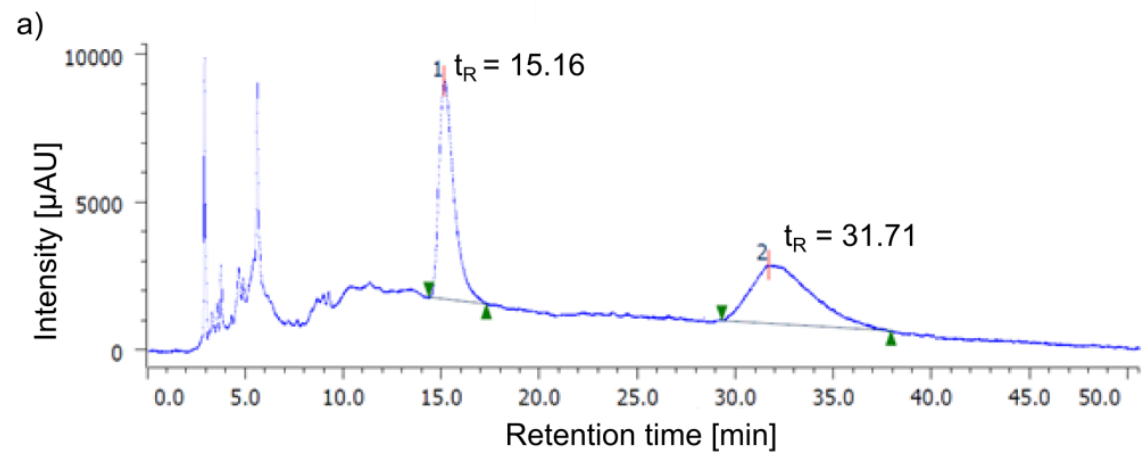

b) $\quad 208.4 \mathrm{~nm}, 9.62087$

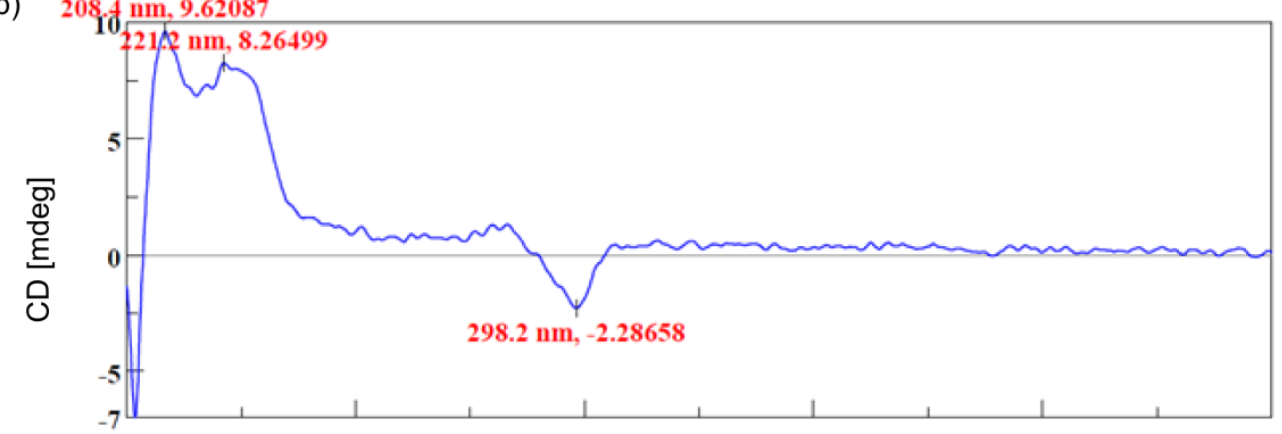

c)

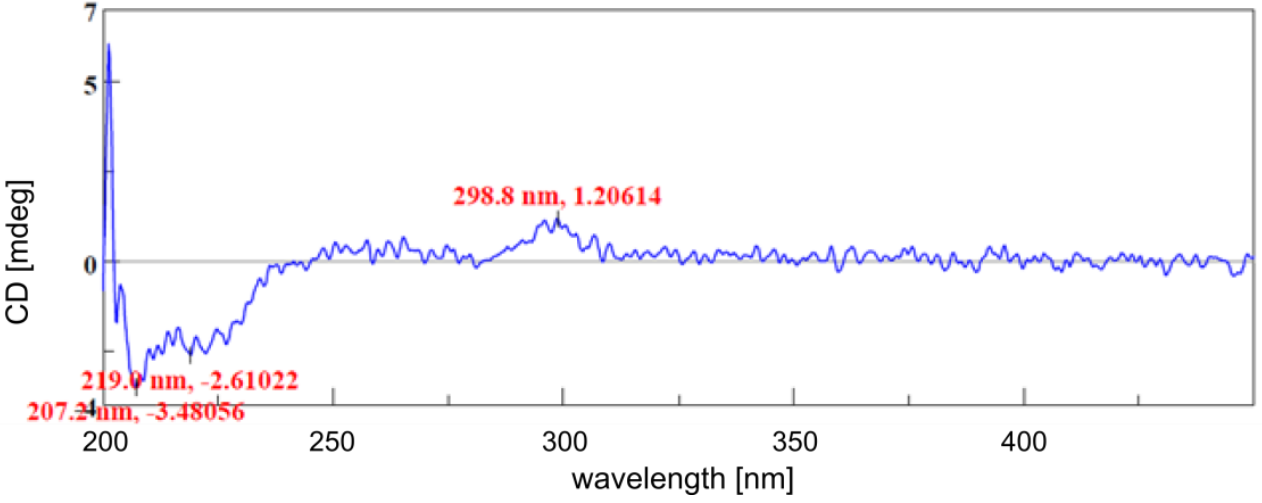

Figure S16. a) Chromatogram of resolution of 4, b) ECD spectrum of first fraction, c) ECD spectrum of second fraction. 


\section{Theoretical calculations}

All calculations were performed within the density functional theory (DFT), MP2 and MP4 approach using Gaussian 09 program suite. ${ }^{1}$ Geometries of 3, 6, 7 and 8 were optimized and energies of HOMO, LUMO orbitals were calculated using different methods: DFT B3LYP/6-311+G(2d,p), DFT B3PW91/6-311+G(2d,p) (also by calculation of ionization energy and electron affinity), MP4/6$311+G(2 d, p)$.

Charge distributions of 3, 6, 7 and 8 were calculated using NBO and MP2/6-311+G(2d,p) method. Fukui functions were calculated as differences between charges on individual atoms in neutral, anionic and cationic forms.

Geometries and energies of transition states were calculated using QST3 and DFT B3LYP/631G(d) method.

Geometries of diastereoisomers and conformers of $\mathbf{4}$ and $\mathbf{5}$ were optimized with the B3LYP functional, employing the $6-31+G(d, p)$ basis set. The geometry of the initial structure of 4 was taken from the crystal structure. Excited electronic states were determined at the B3LYP/6-31G(d) level by means of the time-dependent DFT (TD DFT) approach (100 excited states). The ECD spectrum was simulated by overlapping Gaussian functions for each transition where the width of the band at 1/e height is fixed at $0.16 \mathrm{eV}$ and the resulting intensities of the spectrum were scaled to the experimental values (using the lowest energy bands in UV-VIS spectrum as reference).

Presentation of molecular orbitals was performed by using the GaussView program.

\section{Atomic coordinates for all calculated geometries}

\section{Benzofuran 3}

- $\quad$ DFT B3LYP/6-311+G(2d,p)

Total Energy $=-383.78476487$ a.u.

Cartesian coordinates

$X Y$ $-0.2481410$

$-0.2565630 \quad 0.7521750-0.0000050$

$0.9648940 \quad 1.4341290 \quad-0.0000610$

$\begin{array}{lll}2.1357980 & 0.6907450 & -0.0000550\end{array}$

$\begin{array}{lll}2.1093310 & -0.7125370 & 0.0003260\end{array}$

$\begin{array}{lll}0.9082620 & -1.4113520 & -0.0001570\end{array}$

$\begin{array}{lll}-2.3472740 & -0.0287090 & 0.0001090\end{array}$

$\begin{array}{lll}-1.6487450 & 1.1265810 & 0.0002120\end{array}$

$\begin{array}{lll}0.9972680 & 2.5170820 & -0.0005370\end{array}$

$\begin{array}{lll}3.0919130 & 1.1999980 & -0.0007510\end{array}$

$\begin{array}{lll}3.0428660 & -1.2617490 & 0.0008860\end{array}$

$\begin{array}{lll}0.8723650 & -2.4930630 & 0.0000880\end{array}$

$\begin{array}{lll}-3.4033160 & -0.2398110 & 0.0001430\end{array}$

$\begin{array}{lll}-2.0663260 & 2.1205020 & 0.0003140\end{array}$

$\begin{array}{lll}-1.5300180 & -1.1310450 & -0.0002000\end{array}$

- $\quad$ DFT B3PW91/6-311+G(2d,p)

Total Energy = -383.63231444 a.u.

Cartesian coordinates

$\begin{array}{lll}X & Y & Z \\ 0.0000000 & 0.6941340 & 0.0000000 \\ -0.5099610 & -0.6103580 & 0.0000000 \\ 0.3846990 & -1.6830350 & 0.0000000 \\ 1.7417090 & -1.4081000 & 0.0000000 \\ 2.2192520 & -0.0907640 & 0.0000000 \\ 1.3507240 & 0.9906810 & 0.0000000 \\ -2.1721170 & 0.8698190 & 0.0000000 \\ -1.9398890 & -0.4594820 & 0.0000000 \\ 0.0266870 & -2.7066670 & 0.0000000 \\ 2.4529960 & -2.2265960 & 0.0000000\end{array}$




$\begin{array}{lll}3.2888180 & 0.0869160 & 0.0000000 \\ 1.7050120 & 2.0143910 & 0.0000000 \\ -3.0820740 & 1.4483550 & 0.0000000 \\ -2.6895180 & -1.2354600 & 0.0000000 \\ -1.0185520 & 1.6002120 & 0.0000000\end{array}$

- $\mathrm{MP4} / 6-311+\mathrm{G}(2 \mathrm{~d}, \mathrm{p})$

Total Energy $=-382.74103902$ a.u.

Cartesian coordinates

$\begin{array}{llll} & X & Y & Z \\ C & 0.0000000 & 0.6922050 & 0.0000000 \\ C & -0.5087650 & -0.6080790 & 0.0000000 \\ C & 0.3838780 & -1.6892440 & 0.0000000 \\ C & 1.7452210 & -1.4188460 & 0.0000000 \\ \text { C } & 2.2268070 & -0.0947550 & 0.0000000 \\ \text { C } & 1.3580390 & 0.9898230 & 0.0000000 \\ \text { C } & -2.1799660 & 0.8759280 & 0.0000000 \\ \text { C } & -1.9511780 & -0.4555930 & 0.0000000 \\ \text { H } & 0.0206480 & -2.7123190 & 0.0000000 \\ \text { H } & 2.4551810 & -2.2396520 & 0.0000000 \\ \text { H } & 3.2976230 & 0.0814970 & 0.0000000 \\ \text { H } & 1.7123940 & 2.0146750 & 0.0000000 \\ \text { H } & -3.0895020 & 1.4565240 & 0.0000000 \\ \text { H } & -2.7029910 & -1.2311410 & 0.0000000 \\ \text { O } & -1.0171960 & 1.6102230 & 0.0000000\end{array}$

\section{Diene 6}

- DFT B3LYP/6-311+G(2d,p)

Total Energy $=-420.89246638$ a.u.

Cartesian coordinates

$\begin{array}{llll} & X Y & Z & \\ \text { C } & -0.0615950 & -0.8038570 & -0.0000280 \\ \text { C } & 1.3371080 & -0.2169030 & 0.0000010 \\ \text { C } & 1.4282060 & 1.2373790 & -0.0000170 \\ \text { C } & 0.3116070 & 1.9979640 & 0.0001880 \\ \text { C } & -1.0205080 & 1.4479650 & 0.0001270 \\ \text { C } & -1.2023350 & 0.1076780 & 0.0000580 \\ \text { H } & 2.4200660 & 1.6695660 & -0.0004330 \\ \text { H } & 0.4030260 & 3.0789180 & -0.0002480 \\ \text { H } & -1.8710870 & 2.1206290 & 0.0000550 \\ \text { C } & -0.2115450 & -2.1355650 & 0.0001830 \\ \text { H } & 0.6695230 & -2.7649900 & 0.0003390 \\ \text { H } & -1.1896970 & -2.5963390 & 0.0002550 \\ \text { O } & -2.4223410 & -0.4934680 & -0.0002380 \\ \text { H } & -3.1151760 & 0.1781500 & -0.0001080 \\ \text { O } & 2.3220540 & -0.9432690 & -0.0001290\end{array}$

- $\quad$ DFT B3PW91/6-311+G(2d,p)

Total Energy $=-420.72072554$ a.u.

Cartesian coordinates

$\begin{array}{llll} & X & Y & Z \\ C & -0.0578560 & -0.7993120 & -0.0000410 \\ C & 1.3349160 & -0.2108910 & -0.0000570 \\ C & 1.4207270 & 1.2401610 & 0.0000860 \\ C & 0.3009900 & 1.9946930 & 0.0002040 \\ \text { C } & -1.0261760 & 1.4421120 & 0.0000990 \\ \text { C } & -1.2024220 & 0.1015400 & -0.0000240 \\ \text { H } & 2.4110770 & 1.6782180 & -0.0001710 \\ \text { H } & 0.3888940 & 3.0771150 & -0.0000490 \\ \text { H } & -1.8801470 & 2.1121410 & 0.0000930 \\ \text { C } & -0.1997850 & -2.1308200 & 0.0003290 \\ \text { H } & 0.6884750 & -2.7525200 & 0.0005630 \\ \text { H } & -1.1762150 & -2.5981850 & 0.0004590 \\ \text { O } & -2.4129270 & -0.5037350 & -0.0002650 \\ \text { H } & -3.1049650 & 0.1665790 & -0.0002320 \\ \text { O } & 2.3192410 & -0.9347950 & -0.0002640\end{array}$

- $\mathrm{MP4} / 6-311+\mathrm{G}(2 \mathrm{~d}, \mathrm{p})$

Total Energy $=-419.79182756$ a.u.

Cartesian coordinates

$X Y$ Z

$\begin{array}{llll}C & -0.0737010 & -0.8065330 & -0.0000310 \\ C & 1.3322600 & -0.2430030 & 0.0000060 \\ C & 1.4523960 & 1.2256040 & 0.0000410 \\ C & 0.3518550 & 2.0020840 & -0.0000310 \\ C & -1.0048010 & 1.4626440 & 0.0001460\end{array}$




$\begin{array}{llll}\mathrm{C} & -1.2045980 & 0.1304100 & -0.0000170 \\ \mathrm{H} & 2.4548560 & 1.6386790 & -0.0003400 \\ \mathrm{H} & 0.4584060 & 3.0834790 & -0.0001040 \\ \mathrm{H} & -1.8460740 & 2.1498440 & 0.0000480 \\ \mathrm{C} & -0.2466960 & -2.1365920 & 0.0000410 \\ \mathrm{H} & 0.6220120 & -2.7859520 & 0.0000240 \\ \mathrm{H} & -1.2361670 & -2.5776010 & 0.0001060 \\ \mathrm{O} & -2.4318620 & -0.4657800 & -0.0000790 \\ \mathrm{H} & -3.1116030 & 0.2176140 & -0.0000150 \\ \mathrm{O} & 2.3091460 & -0.9759380 & -0.0000020\end{array}$

\section{$\alpha$-methylstyren 7}

- DFT B3LYP/6-311+G(2d,p)

Total Energy $=-349.06609530$ a.u.

Cartesian coordinates

$\begin{array}{llll} & X Y & Z & \\ \text { C } & -0.2053510 & 0.0529610 & 0.0117110 \\ \text { C } & 0.4682190 & -1.1561130 & 0.2282220 \\ \text { C } & 1.8574360 & -1.2156600 & 0.2304650 \\ \text { C } & 2.6091300 & -0.0692270 & 0.0012620 \\ \text { C } & 1.9564010 & 1.1373880 & -0.2345430 \\ \text { C } & 0.5694500 & 1.1957430 & -0.2327060 \\ \text { H } & -0.0948030 & -2.0620990 & 0.4127110 \\ \text { H } & 2.3525880 & -2.1626750 & 0.4111160 \\ \text { H } & 3.6915600 & -0.1160110 & -0.0037200 \\ \text { H } & 2.5302920 & 2.0352360 & -0.4326530 \\ \text { H } & 0.0761180 & 2.1355960 & -0.4471380 \\ \text { C } & -1.6904100 & 0.1207800 & 0.0329760 \\ \text { C } & -2.3367060 & 1.2168550 & 0.4414510 \\ \text { H } & -1.8141160 & 2.0908490 & 0.8091050 \\ \text { H } & -3.4190820 & 1.2626800 & 0.4365920 \\ \text { C } & -2.4553520 & -1.1015070 & -0.4128950 \\ \text { H } & -2.1269770 & -1.4357660 & -1.4009470 \\ \text { H } & -2.3076380 & -1.9406620 & 0.2730620 \\ \text { H } & -3.5248500 & -0.8944580 & -0.4537900\end{array}$

- $\quad$ DFT B3PW91/6-311+G(2d,p)

Total Energy $=-348.92419856$ a.u.

Cartesian coordinates

\begin{tabular}{|c|c|c|c|}
\hline & $X Y$ & Z & \\
\hline C & -0.2057790 & 0.0525100 & 0.0116040 \\
\hline C & 0.4661620 & -1.1550450 & 0.2253130 \\
\hline C & 1.8532110 & -1.2142040 & 0.2279550 \\
\hline C & 2.6033630 & -0.0685540 & 0.0017940 \\
\hline C & 1.9514870 & 1.1366240 & -0.2317140 \\
\hline C & 0.5666250 & 1.1942830 & -0.2303600 \\
\hline $\mathrm{H}$ & -0.0987340 & -2.0619540 & 0.4073490 \\
\hline $\mathrm{H}$ & 2.3492470 & -2.1623260 & 0.4067580 \\
\hline $\mathrm{H}$ & 3.6868790 & -0.1150690 & -0.0030850 \\
\hline $\mathrm{H}$ & 2.5257860 & 2.0357570 & -0.4286460 \\
\hline $\mathrm{H}$ & 0.0707220 & 2.1343250 & -0.4449220 \\
\hline C & -1.6865000 & 0.1193270 & 0.0314750 \\
\hline C & -2.3307290 & 1.2168450 & 0.4366780 \\
\hline $\mathrm{H}$ & -1.8046330 & 2.0907290 & 0.8032020 \\
\hline $\mathrm{H}$ & -3.4141860 & 1.2633090 & 0.4317400 \\
\hline C & -2.4477280 & -1.1007000 & -0.4079340 \\
\hline $\mathrm{H}$ & -2.1129910 & -1.4441130 & -1.3912690 \\
\hline $\mathrm{H}$ & -2.3054190 & -1.9343200 & 0.2870150 \\
\hline $\mathrm{H}$ & -3.5173380 & -0.8928520 & -0.4570040 \\
\hline
\end{tabular}

- $\mathrm{MP4} / 6-311+\mathrm{G}(2 \mathrm{~d}, \mathrm{p})$

Total Energy $=-348.03079305$ a.u.

Cartesian coordinates

$\begin{array}{llll} & X Y & Z & \\ C & -0.2041350 & 0.0486220 & 0.0164600 \\ \text { C } & 0.4693100 & -1.1516490 & 0.2761880 \\ \text { C } & 1.8619820 & -1.2067660 & 0.2727340 \\ \text { C } & 2.6092610 & -0.0634180 & -0.0042800 \\ \text { C } & 1.9508510 & 1.1343000 & -0.2810110 \\ \text { C } & 0.5590420 & 1.1872630 & -0.2735860 \\ \text { H } & -0.0949680 & -2.0515700 & 0.4991690 \\ \text { H } & 2.3632980 & -2.1457570 & 0.4872720 \\ \text { H } & 3.6938460 & -0.1070600 & -0.0129990 \\ \text { H } & 2.5219420 & 2.0279220 & -0.5142440 \\ \text { H } & 0.0541750 & 2.1174520 & -0.5155910 \\ \text { C } & -1.6934430 & 0.1143440 & 0.0418670 \\ \text { C } & -2.3313250 & 1.1828370 & 0.5371560\end{array}$




$\begin{array}{lll}-1.7924300 & 2.0208170 & 0.9670380 \\ -3.4154510 & 1.2382630 & 0.5386700 \\ -2.4531300 & -1.0715870 & -0.5045830 \\ -2.1160450 & -1.3172240 & -1.5168260 \\ -2.2992670 & -1.9618830 & 0.1138510 \\ -3.5255690 & -0.8646420 & -0.5320090\end{array}$

\section{indene 8}

- $\quad$ DFT B3LYP/6-311+G(2d,p)

Total Energy $=-347.86056641$ a.u.

Cartesian coordinates

$\begin{array}{lll}X & Y & Z \\ 0.4276940 & 0.6164140 & 0.0000000 \\ 0.0000000 & -0.7243210 & 0.0000000 \\ -1.3491580 & -1.0326750 & 0.0000000 \\ -2.2795660 & 0.0092870 & 0.0000000 \\ -1.8573620 & 1.3378090 & 0.0000000 \\ -0.5002490 & 1.6537490 & 0.0000000 \\ 1.8915820 & 0.6278560 & 0.0000000 \\ 2.3546660 & -0.6320810 & 0.0000000 \\ 1.2151150 & -1.6202860 & 0.0000000 \\ -1.6868370 & -2.0635270 & 0.0000000 \\ -3.3392540 & -0.2169410 & 0.0000000 \\ -2.5935900 & 2.1330870 & 0.0000000 \\ -0.1776140 & 2.6888600 & 0.0000000 \\ 2.4964270 & 1.5253930 & 0.0000000 \\ 3.3954600 & -0.9256890 & 0.0000000 \\ 1.2445310 & -2.2778510 & 0.8770020 \\ 1.2445310 & -2.2778510 & -0.8770020\end{array}$

- $\quad$ DFT B3PW91/6-311+G(2d,p)

Total Energy = -347.72331470 a.u.

Cartesian coordinates

$\begin{array}{lll}X & Y & Z \\ 0.4290580 & 0.6151110 & 0.0000000 \\ 0.0000000 & -0.7227160 & 0.0000000 \\ -1.3481150 & -1.0289460 & 0.0000000 \\ -2.2747310 & 0.0127940 & 0.0000000 \\ -1.8510540 & 1.3387980 & 0.0000000 \\ -0.4960730 & 1.6527120 & 0.0000000 \\ 1.8891630 & 0.6232750 & 0.0000000 \\ 2.3472420 & -0.6377820 & 0.0000000 \\ 1.2085560 & -1.6173410 & 0.0000000 \\ -1.6875570 & -2.0602540 & 0.0000000 \\ -3.3359090 & -0.2116230 & 0.0000000 \\ -2.5870310 & 2.1357780 & 0.0000000 \\ -0.1716540 & 2.6882950 & 0.0000000 \\ 2.4981890 & 1.5191440 & 0.0000000 \\ 3.3882110 & -0.9349130 & 0.0000000 \\ 1.2357370 & -2.2759270 & 0.8771850 \\ 1.2357370 & -2.2759270 & -0.8771850\end{array}$

- $\quad \mathrm{MP} 4 / 6-311+\mathrm{G}(2 \mathrm{~d}, \mathrm{p})$

Total Energy $=-346.84733088$ a.u.

Cartesian coordinates

$\mathrm{X}$

$\begin{array}{lll} & Y & Z \\ 0.2088980 & 0.7187840 & 0.00\end{array}$

$\begin{array}{lll}0.2286720 & -0.6861370 & 0.0000000\end{array}$

$\begin{array}{lll}-0.9556850 & -1.4079700 & 0.0000000\end{array}$

$\begin{array}{lll}-2.1695620 & -0.7112440 & 0.0000000\end{array}$

$\begin{array}{lll}-2.1883850 & 0.6852420 & 0.0000000\end{array}$

$\begin{array}{lll}-0.9964520 & 1.4144410 & 0.0000000\end{array}$

$\begin{array}{lll}1.6029020 & 1.1964150 & 0.0000000\end{array}$

$\begin{array}{lll}2.4381150 & 0.1420200 & 0.0000000\end{array}$

$\begin{array}{lll}1.6649920 & -1.1595690 & 0.0000000\end{array}$

$\begin{array}{lll}-0.9485680 & -2.4948150 & 0.0000000\end{array}$

$\begin{array}{lll}-3.1057200 & -1.2610280 & 0.0000000\end{array}$

$\begin{array}{lll}-3.1397200 & 1.2085110 & 0.0000000\end{array}$

$\begin{array}{lll}-1.0147020 & 2.5007000 & 0.0000000\end{array}$

$\begin{array}{lll}1.8941620 & 2.2411060 & 0.0000000\end{array}$

$\begin{array}{lll}3.5209190 & 0.1922270 & 0.0000000\end{array}$

$\begin{array}{lll}1.8963340 & -1.7692920 & 0.8819840\end{array}$

$\begin{array}{lll}1.8963340 & -1.7692920 & -0.8819840\end{array}$

\section{TS-endo-"4"}

- $\quad$ QST3 DFT B3LYP/631G(d) 
Total energy $=-804.40848620 \mathrm{a} . \mathrm{u}$.

One imaginary frequency

Cartesian coordinates

\begin{tabular}{|c|c|c|c|}
\hline & $\mathrm{x}$ & $Y$ & Z \\
\hline C & -1.5428300 & -0.5297970 & -0.8416370 \\
\hline C & -1.7952120 & -0.4518560 & 0.5491790 \\
\hline C & -2.4957810 & 0.6632120 & 1.0572120 \\
\hline C & -2.9303670 & 1.6291770 & 0.1655300 \\
\hline C & -2.6680380 & 1.5182280 & -1.2199650 \\
\hline C & -1.9664380 & 0.4402740 & -1.7481030 \\
\hline C & -0.5526200 & -2.2780810 & 0.0623710 \\
\hline C & -1.2087730 & -1.6100870 & 1.1159770 \\
\hline $\mathrm{H}$ & -2.6791360 & 0.7567550 & 2.1229820 \\
\hline $\mathrm{H}$ & -3.4723020 & 2.4961150 & 0.5318540 \\
\hline $\mathrm{H}$ & -3.0214860 & 2.2984890 & -1.8882430 \\
\hline $\mathrm{H}$ & -1.7568760 & 0.3478260 & -2.8082490 \\
\hline $\mathrm{H}$ & -0.4271440 & -3.3507510 & -0.0223030 \\
\hline $\mathrm{H}$ & -1.2273750 & -1.9206440 & 2.1481480 \\
\hline $\mathrm{O}$ & -0.8849100 & -1.6735460 & -1.1567250 \\
\hline C & 1.4568130 & 1.6649380 & 1.4446460 \\
\hline C & 1.2517110 & 0.2335430 & 1.4545150 \\
\hline C & 1.6365000 & -0.5088920 & 0.2441220 \\
\hline C & 2.1323830 & 0.1989270 & -0.8940740 \\
\hline C & 2.3353020 & 1.5594150 & -0.8393920 \\
\hline C & 1.9916800 & 2.2770070 & 0.3432950 \\
\hline $\mathrm{H}$ & 1.1825030 & 2.2197170 & 2.3360270 \\
\hline $\mathrm{H}$ & 2.7569620 & 2.0882390 & -1.6916030 \\
\hline $\mathrm{H}$ & 2.1587490 & 3.3520290 & 0.3525810 \\
\hline C & 1.4107300 & -1.8946880 & 0.2569080 \\
\hline $\mathrm{H}$ & 1.7568630 & -2.4780130 & -0.5912490 \\
\hline $\mathrm{H}$ & 1.4679940 & -2.3746460 & 1.2291020 \\
\hline $\mathrm{O}$ & 2.4294030 & -0.5563130 & -1.9970280 \\
\hline $\mathrm{H}$ & 2.7801090 & 0.0298180 & -2.6858420 \\
\hline 0 & 0.7243540 & -0.3867480 & 2.4116660 \\
\hline
\end{tabular}

\section{TS-exo-"4"}

- $\quad$ QST3 DFT B3LYP/631G(d)

Total energy $=-804.40763128$ a.u.

One imaginary frequency

Cartesian coordinates

$\begin{array}{lll}X & Y & Z \\ -2.5677530 & -0.8413980 & 0.1305640 \\ -2.2055280 & 0.4598300 & 0.5519800 \\ -3.1272010 & 1.5149420 & 0.3937920 \\ -4.3629480 & 1.2292990 & -0.1653610 \\ -4.6933930 & -0.0804930 & -0.5794540 \\ -3.7986380 & -1.1393400 & -0.4467690 \\ -0.4765010 & -0.9810250 & 0.8477780 \\ -0.8858590 & 0.3512820 & 1.0609510 \\ -2.8647900 & 2.5242850 & 0.6947980 \\ -5.0907930 & 2.0242390 & -0.2983120 \\ -5.6711950 & -0.2661490 & -1.0148870 \\ -4.0429990 & -2.1471140 & -0.7649090 \\ 0.1698860 & -1.5611190 & 1.4937340 \\ -0.2935480 & 1.1331390 & 1.5066290 \\ -1.5671050 & -1.7284110 & 0.3708810 \\ 3.0083440 & 1.9594870 & -0.0825590 \\ 1.8052000 & 1.2204880 & -0.4002280 \\ 1.9142110 & -0.2431070 & -0.4569630 \\ 3.1473360 & -0.8741870 & -0.1095990 \\ 4.2673360 & -0.1257050 & 0.1728170 \\ 4.1786250 & 1.2976450 & 0.1731640 \\ 2.9425560 & 3.0424910 & -0.0585910 \\ 5.2149370 & -0.6112930 & 0.3958420 \\ 5.0800500 & 1.8638260 & 0.3977830 \\ 0.7353510 & -0.9603280 & -0.7291740 \\ 0.0030090 & -0.4556790 & -1.3519750 \\ 0.8040430 & -2.0370510 & -0.8530640 \\ 3.1432200 & -2.2455260 & -0.1014870 \\ 4.0371860 & -2.5552640 & 0.1131460 \\ 0.6839080 & 1.7641050 & -0.5726230\end{array}$

\section{TS-exo-“5”}

- $\quad$ QST3 DFT B3LYP/631G(d)

Total energy $=-804.40455736$ a.u. 
One imaginary frequency

Cartesian coordinates

$Y \quad Z$

$\begin{array}{lll}-2.6078070 & -0.7836920 & 0.1549600\end{array}$

$\begin{array}{lll}-2.0655250 & 0.4576630 & 0.5095000\end{array}$

$\begin{array}{lll}-2.8276860 & 1.6093620 & 0.2962150\end{array}$

$\begin{array}{lll}-4.1046380 & 1.4695490 & -0.2519300\end{array}$

$\begin{array}{lll}-4.6131830 & 0.2091960 & -0.6024370\end{array}$

$\begin{array}{lll}-3.8631930 & -0.9535840 & -0.4057090\end{array}$

$\begin{array}{lll}-0.6172460 & -1.2253630 & 0.9814450\end{array}$

$\begin{array}{lll}-0.6987730 & 0.1849800 & 0.9726390\end{array}$

$\begin{array}{lll}-2.4410200 & 2.5912140 & 0.5550460\end{array}$

$\begin{array}{lll}-4.7159180 & 2.3528880 & -0.4131430\end{array}$

$\begin{array}{lll}-5.6077560 & 0.1334410 & -1.0321260\end{array}$

$\begin{array}{lll}-4.2379510 & -1.9371450 & -0.6678500\end{array}$

$\begin{array}{lll}0.0652800 & -1.8931170 & 1.4763790\end{array}$

$\begin{array}{lll}-0.1701930 & 0.7905760 & 1.6972110\end{array}$

$\begin{array}{lll}-1.7239610 & -1.8021190 & 0.4735490\end{array}$

$\begin{array}{lll}3.3375840 & -1.5726630 & -0.1969300\end{array}$

$\begin{array}{lll}2.0011070 & -1.0950500 & -0.4375660\end{array}$

$\begin{array}{lll}1.7856370 & 0.3462590 & -0.4218370\end{array}$

$\begin{array}{lll}2.8582370 & 1.2187290 & -0.0690090\end{array}$

$\begin{array}{lll}4.1266270 & 0.7212850 & 0.1374980\end{array}$

$\begin{array}{lll}4.3491440 & -0.6813820 & 0.0588580\end{array}$

$\begin{array}{lll}3.5096070 & -2.6437120 & -0.2280330\end{array}$

$\begin{array}{lll}4.9543110 & 1.3925960 & 0.3562920\end{array}$

$\begin{array}{lll}5.3601040 & -1.0481710 & 0.2225370\end{array}$

$\begin{array}{lll}0.4645690 & 0.7753060 & -0.6309860\end{array}$

$\begin{array}{lll}0.2434350 & 1.8380370 & -0.6065520\end{array}$

$\begin{array}{lll}-0.1344640 & 0.1797590 & -1.3096950\end{array}$

$\begin{array}{lll}2.5560470 & 2.5532050 & 0.0100350\end{array}$

$\begin{array}{lll}3.3682290 & 3.0405960 & 0.2197500\end{array}$

$\begin{array}{lll}1.0000660 & -1.8611520 & -0.5883440\end{array}$

\section{TS-endo-"5"}

- $\quad$ QST3 DFT B3LYP/631G(d)

Total energy $=-804.40419260 \mathrm{a} . \mathrm{u}$.

One imaginary frequency

Cartesian coordinates

$\begin{array}{llll} & X & Y & Z \\ \text { C } & -1.6373120 & -0.6920130 & -0.7237620 \\ \text { C } & -1.7331560 & -0.3875080 & 0.6381470 \\ \text { C } & -2.4504910 & 0.7478360 & 1.0232540 \\ \text { C } & -3.0546280 & 1.5217190 & 0.0304520 \\ \text { C } & -2.9328350 & 1.1882390 & -1.3276330 \\ \text { C } & -2.2102040 & 0.0635730 & -1.7339690 \\ \text { C } & -0.5399320 & -2.2964660 & 0.3153670 \\ \text { C } & -0.9289820 & -1.3973650 & 1.3332570 \\ \text { H } & -2.5395830 & 1.0238830 & 2.0701900 \\ \text { H } & -3.6267730 & 2.4008770 & 0.3130730 \\ \text { H } & -3.4096030 & 1.8126730 & -2.0776950 \\ \text { H } & -2.1044280 & -0.2127490 & -2.7774660 \\ \text { H } & -0.1842640 & -3.3102970 & 0.3665750 \\ \text { H } & -1.1306590 & -1.7431940 & 2.3399980 \\ \text { O } & -0.9289420 & -1.8708880 & -0.9050390 \\ \text { C } & 2.5800810 & -0.3962640 & -1.3719360 \\ \text { C } & 1.9612450 & -0.8996630 & -0.1753720 \\ \text { C } & 1.4845060 & 0.0701880 & 0.8034240 \\ \text { C } & 1.5644340 & 1.4649190 & 0.5143120 \\ \text { C } & 2.1878440 & 1.9048590 & -0.6332680 \\ \text { C } & 2.6975750 & 0.9575240 & -1.5633530 \\ \text { H } & 2.9462390 & -1.1146920 & -2.0979530 \\ \text { H } & 2.2945160 & 2.9698560 & -0.8284510 \\ \text { H } & 3.1838210 & 1.3336890 & -2.4607970 \\ \text { C } & 0.8364460 & -0.4606050 & 1.9284700 \\ \text { H } & 1.2083460 & -1.4109530 & 2.2942870 \\ \text { H } & 0.4261110 & 0.2182240 & 2.6698500 \\ \text { O } & 1.0381880 & 2.3125190 & 1.4524820 \\ \text { H } & 1.1479590 & 3.2239750 & 1.1391700 \\ \text { O } & 1.7458520 & -2.1322730 & 0.0406690\end{array}$

\section{TS-endo-"9"}

- $\quad$ QST3 DFT B3LYP/631G(d)

Total energy $=-768.50330271$ a.u.

One imaginary frequency

Cartesian coordinates 


$\begin{array}{lll}-1.5192420 & -0.6091150 & -0.9117310 \\ -1.7827480 & -0.5037000 & 0.4749810 \\ -2.5160710 & 0.5822740 & 0.9814330 \\ -2.9851800 & 1.5416430 & 0.0915300 \\ -2.7179370 & 1.4365460 & -1.2838220 \\ -1.9800370 & 0.3649440 & -1.7925000 \\ -0.4380340 & -2.3759690 & 0.2321160 \\ -1.1709660 & -1.6196900 & 1.1485690 \\ -2.6955160 & 0.6738840 & 2.0485850 \\ -3.5542760 & 2.3899130 & 0.4620290 \\ -3.0896350 & 2.2017550 & -1.9601950 \\ -1.7793400 & 0.2937030 & -2.8587300 \\ -0.1967800 & -3.4185310 & 0.4160870 \\ -1.2845020 & -1.8388620 & 2.2012830 \\ 1.2434480 & 1.8632200 & 1.2803340 \\ 1.1306520 & 0.4304670 & 1.4454580 \\ 1.6704100 & -0.4138230 & 0.3663930 \\ 2.2032780 & 0.1985230 & -0.8108650 \\ 2.3121870 & 1.5656390 & -0.9046090 \\ 1.8281430 & 2.3848320 & 0.1586850 \\ 0.8585820 & 2.4937870 & 2.0751460 \\ 2.7583100 & 2.0253190 & -1.7840510 \\ 1.9227640 & 3.4634130 & 0.0526120 \\ 1.5490080 & -1.7953980 & 0.5398610 \\ 1.9906630 & -2.4616270 & -0.1945950 \\ 1.5184180 & -2.1467880 & 1.5645900 \\ 2.6194040 & -0.6553480 & -1.8029210 \\ 2.9873670 & -0.1263570 & -2.5283390 \\ 0.5637440 & -0.1151840 & 2.4240680 \\ -0.7620790 & -1.8869020 & -1.1771510 \\ -1.4069060 & -2.6227450 & -1.6812500 \\ 0.1166650 & -1.7435440 & -1.8144460\end{array}$

\section{TS-exo-“9”}

- $\quad$ QST3 DFT B3LYP/631G(d)

Total energy $=-768.50097595$ a.u.

One imaginary frequency

Cartesian coordinates

\begin{tabular}{lll}
$X$ & $Y$ & $Z$ \\
2.6510960 & 0.8983780 & 0.1130000 \\
2.1781550 & -0.3549190 & 0.5663560 \\
2.9455270 & -1.5150600 & 0.3930350 \\
4.1837460 & -1.4051780 & -0.2345210 \\
4.6475120 & -0.1625450 & -0.6916860 \\
3.8816410 & 0.9969230 & -0.5259920 \\
0.4507720 & 1.1462230 & 0.9773100 \\
0.8722930 & -0.1722230 & 1.1555830 \\
2.5694900 & -2.4782590 & 0.7247090 \\
4.7944700 & -2.2915300 & -0.3829120 \\
5.6151110 & -0.1002910 & -1.1825610 \\
4.2520370 & 1.9543300 & -0.8845960 \\
-0.3023740 & 1.6041420 & 1.6089370 \\
0.3407700 & -0.9389820 & 1.7001300 \\
-2.8337190 & -2.0200050 & -0.0253360 \\
-1.6849210 & -1.1946340 & -0.3269930 \\
-1.9109100 & 0.2509290 & -0.4544440 \\
-3.2100040 & 0.7889320 & -0.1873120 \\
-4.2731010 & -0.0405360 & 0.0825530 \\
-4.0653250 & -1.4501400 & 0.1515820 \\
-2.6789390 & -3.0914210 & 0.0513690 \\
-5.2677860 & 0.3699860 & 0.2426580 \\
-4.9253860 & -2.0814090 & 0.3646580 \\
-0.7911920 & 1.0474520 & -0.7106440 \\
0.0164480 & 0.5940980 & -1.2753980 \\
-0.9311990 & 2.1174190 & -0.8277430 \\
-3.3236570 & 2.1533190 & -0.2497940 \\
-4.2487360 & 2.3928490 & -0.0822440 \\
-0.5140910 & -1.6459250 & -0.4223510 \\
1.6356390 & 1.9624730 & 0.4582300 \\
2.0070460 & 2.6143210 & 1.2627790 \\
1.3977560 & 2.6191840 & -0.3869630 \\
& & \\
\hline
\end{tabular}

\section{TS-exo-"10"}

- $\quad$ QST3 DFT B3LYP/631G(d)

Total energy $=-768.49603555$ a.u.

One imaginary frequency

Cartesian coordinates 
$\mathrm{X}$

$-26668110-0.8241690$

$\begin{array}{lll}-2.7886670 & 1.5875950 & 0.3928850\end{array}$

$\begin{array}{lll}-4.0658330 & 1.5689910 & -0.1759000\end{array}$

$\begin{array}{lll}-4.6308600 & 0.3702400 & -0.6217280\end{array}$

$\begin{array}{lll}-3.9306110 & -0.8360200 & -0.4956020\end{array}$

$\begin{array}{lll}-0.5356620 & -1.2750520 & 1.0087960\end{array}$

$\begin{array}{lll}-0.7297610 & 0.1110630 & 1.0341450\end{array}$

$\begin{array}{lll}-2.3515080 & 2.5238500 & 0.7319320\end{array}$

$\begin{array}{lll}-4.6237520 & 2.4965200 & -0.2745310\end{array}$

$\begin{array}{lll}-5.6236040 & 0.3734190 & -1.0637040\end{array}$

$\begin{array}{lll}-4.3787620 & -1.7669740 & -0.8352540\end{array}$

$\begin{array}{lll}0.1914520 & -1.8066460 & 1.6042140\end{array}$

$\begin{array}{lll}-0.2288410 & 0.7611960 & 1.7429160\end{array}$

$\begin{array}{lll}3.3181000 & -1.5636900 & -0.1956950\end{array}$

$\begin{array}{lll}1.9903040 & -1.0653360 & -0.4299840\end{array}$

$\begin{array}{lll}1.7879930 & 0.3771390 & -0.4164340\end{array}$

$\begin{array}{lll}2.8804810 & 1.2367800 & -0.0788770\end{array}$

$\begin{array}{lll}4.1419940 & 0.7202040 & 0.1144500\end{array}$

$\begin{array}{lll}4.3456420 & -0.6861330 & 0.0417880\end{array}$

$\begin{array}{lll}3.4728410 & -2.6374140 & -0.2214860\end{array}$

$\begin{array}{lll}4.9822110 & 1.3803310 & 0.3192440\end{array}$

$\begin{array}{lll}5.3534160 & -1.0654120 & 0.1950640\end{array}$

$\begin{array}{lll}0.4761100 & 0.8205350 & -0.6044700\end{array}$

$\begin{array}{lll}0.2480360 & 1.8789830 & -0.5298450\end{array}$

$\begin{array}{lll}-0.1582900 & 0.2347360 & -1.2565340\end{array}$

$\begin{array}{lll}2.5986210 & 2.5740770-0.0076830\end{array}$

$\begin{array}{lll}3.4167530 & 3.0521150 & 0.2007120\end{array}$

$\begin{array}{lll}0.9746990 & -1.8194760 & -0.5612930\end{array}$

$-1.7195710 \quad-1.9667630 \quad 0.3841320$

$\begin{array}{lll}-2.1580620 & -2.6915910 & 1.0861180\end{array}$

$\begin{array}{lll}-1.4165620 & -2.5338690 & -0.5046700\end{array}$

\section{TS-endo-“10”}

- $\quad$ QST3 DFT B3LYP/631G(d)

Total energy $=-768.49470711$ a.u.

One imaginary frequency

Cartesian coordinates

1.7650300

$\begin{array}{lll}1.6847250 & -0.2675140 & -0.6585980\end{array}$

$\begin{array}{lll}2.2659870 & 0.9431280 & -1.0411140\end{array}$

$\begin{array}{lll}2.9259270 & 1.7083940 & -0.0767260\end{array}$

$\begin{array}{lll}2.9956010 & 1.2768910 & 1.2526750\end{array}$

$\begin{array}{lll}2.4115510 & 0.0650440 & 1.6371680\end{array}$

$\begin{array}{lll}0.5704700 & -2.3113670 & -0.5896680\end{array}$

$0.9181380 \quad-1.2567790 \quad-1.4435810$

$\begin{array}{lll}2.2047640 & 1.2899510 & -2.0697200\end{array}$

$\begin{array}{lll}3.3873760 & 2.6505340 & -0.3613920\end{array}$

$\begin{array}{lll}3.5105920 & 1.8857270 & 1.9910710\end{array}$

$\begin{array}{lll}2.4741730 & -0.2698930 & 2.6699970\end{array}$

$\begin{array}{lll}0.2621290 & -3.2919090 & -0.9219030\end{array}$

$\begin{array}{lll}1.0723730 & -1.3999060 & -2.5083590\end{array}$

$\begin{array}{lll}-2.3984490 & -0.5656930 & 1.4757570\end{array}$

$\begin{array}{lll}-1.8776130 & -0.9644730 & 0.1968420\end{array}$

$\begin{array}{lll}-1.5621870 & 0.0784670 & -0.7717560\end{array}$

$\begin{array}{lll}-1.7017650 & 1.4505890 & -0.3962310\end{array}$

$\begin{array}{lll}-2.2361600 & 1.7881050 & 0.8275230\end{array}$

$\begin{array}{lll}-2.5884310 & 0.7656200 & 1.7504560\end{array}$

$\begin{array}{lll}-2.6511060 & -1.3428570 & 2.1899450\end{array}$

$\begin{array}{lll}-2.3911720 & 2.8323780 & 1.0903150\end{array}$

$\begin{array}{lll}-3.0104870 & 1.0634440 & 2.7075860\end{array}$

$-0.9818590 \quad-0.3477520 \quad-1.9669580$

$\begin{array}{lll}-1.2838410 & -1.3150950 & -2.3482190\end{array}$

$\begin{array}{lll}-0.6441300 & 0.3861530 & -2.6916310\end{array}$

$\begin{array}{lll}-1.3277000 & 2.3752980 & -1.3317280\end{array}$

$\begin{array}{lll}-1.4458000 & 3.2614720 & -0.9551200\end{array}$

$\begin{array}{lll}-1.6018860 & -2.1701550 & -0.0998810\end{array}$

$\begin{array}{lll}1.1069160 & -2.0634260 & 0.8001530\end{array}$

$\begin{array}{lll}1.8391260 & -2.8448640 & 1.0575060\end{array}$

$\begin{array}{lll}0.3254090 & -2.1075710 & 1.5672710\end{array}$

Benzofuran resorcinarene derivative out-exo and in-endo (all- $R, R$, all- $R$ )-4

- $\quad$ DFT B3LYP/6-311+G(d,p)

Total Energy = -3999.66201976 a.u.

Cartesian coordinates 


\begin{tabular}{|c|c|c|}
\hline & 2.4890490 & \\
\hline & 1.8481830 & 2.4773620 \\
\hline 2723320 & 2537690 & 1028770 \\
\hline .0010910 & .0665300 & \\
\hline 2990990 & 1327990 & -0.54617 \\
\hline 3097890 & 3.3776570 & 0.0516240 \\
\hline 9426220 & 8390390 & -0.638436 \\
\hline 8384750 & 4552670 & -0.57236 \\
\hline .5774480 & .4520830 & -0.52261 \\
\hline .0283840 & 8160080 & -0.766872 \\
\hline 1956530 & .2657840 & 0.218690 \\
\hline 3.0004390 & 5.6411180 & -1.592971 \\
\hline-2.8524970 & 6.6180210 & -1.12070 \\
\hline-1.6648550 & 9151450 & -1.78295 \\
\hline-1.7933940 & 1.2199260 & -2.62331 \\
\hline .8751030 & .6152680 & -205580 \\
\hline .6107660 & 984110 & -2.89140 \\
\hline .8996140 & & -2.85078 \\
\hline .2386800 & & -1.6 \\
\hline-6.5231540 & & \\
\hline 6.7923870 & & -0.53451 \\
\hline .4585420 & & -2.49359 \\
\hline-8.4639630 & & -2.36769 \\
\hline-7.0995840 & 77770 & -3.69157 \\
\hline-7.8358140 & & 4247 \\
\hline-5.813 & & -3. \\
\hline-5.53 & & -4. \\
\hline-3.22 & & 1.7 \\
\hline-4.1556420 & & 1.422369 \\
\hline-3.2341190 & & 3.268416 \\
\hline-2.2966470 & & 5548 \\
\hline 1.02 & & 0030 \\
\hline 600 & & 25320 \\
\hline-2.7076 & & 2628 \\
\hline-4.85 & & 6270 \\
\hline-5.00 & & 1090 \\
\hline-4.98 & & 3050 \\
\hline-5.6420770 & & 1771 \\
\hline-3.2477290 & & 5.478523 \\
\hline-3.970727 & & 5.9 \\
\hline .3730250 & & 457 \\
\hline-2.2426930 & & 074 \\
\hline 1.676 & & 449 \\
\hline 2.52 & & 7330 \\
\hline 2.1592320 & & 2369 \\
\hline 1.6408760 & & 68416 \\
\hline 4890490 & & 27890 \\
\hline 8481830 & & 362 \\
\hline 3776570 & & 624 \\
\hline & & 548 \\
\hline 0 & & 030 \\
\hline & & 32 \\
\hline .70 & & 528 \\
\hline 3.5892580 & & 627 \\
\hline 3.7758480 & & 409 \\
\hline 1053230 & & \\
\hline 2.9091240 & & 771 \\
\hline 2.858 & & 523 \\
\hline 2.155 & 270 & 569 \\
\hline 3.8203 & 250 & 457 \\
\hline 2.487 & 930 & 074 \\
\hline & 3320 & \\
\hline 4.0665300 & & \\
\hline ח & 2990990 & \\
\hline 8390390 & 0.9426220 & \\
\hline 4520830 & 3.5774480 & -0.522615 \\
\hline 8160080 & 3840 & -0.76687 \\
\hline & & 0.218690 \\
\hline & & 150207 \\
\hline 6180210 & & -1.12070 \\
\hline & .6648550 & -1.78295 \\
\hline 4.2199260 & 7933940 & -2.62331 \\
\hline 5.6152680 & 8751030 & -2.05580 \\
\hline 4.823 & & \\
\hline 2.0886460 & 8730 & \\
\hline 0.776 & & 1.627890 \\
\hline & & \\
\hline 2.3097890 & -3.3776570 & 0.051624 \\
\hline 4.4552670 & -1.8384750 & -0.57236 \\
\hline 3.5774480 & -3.4520830 & \\
\hline & & 7 \\
\hline
\end{tabular}

\begin{tabular}{|c|c|c|}
\hline 4.1556420 & -2.1592320 & 1.4223690 \\
\hline 3.2341190 & -1.6408760 & 3.2684160 \\
\hline 2.2966470 & -1.2155940 & 3.6455480 \\
\hline 4.0257460 & -0.9470010 & 3.5820030 \\
\hline 3.4600600 & -3.0004390 & 3.9625320 \\
\hline 5.8984110 & 3.6107660 & -2.8914080 \\
\hline 5.4543840 & 4.8996140 & -2.8507820 \\
\hline 4.3188250 & 6.5231540 & -1.4613960 \\
\hline 5.6009650 & 5.8138630 & -3.8919810 \\
\hline 3.8204370 & 6.7923870 & -0.5345100 \\
\hline 4.4534420 & 7.4585420 & -2.4935950 \\
\hline 4.0648580 & 8.4639630 & -2.3676940 \\
\hline 5.0877770 & 7.0995840 & -3.6915710 \\
\hline 5.1862040 & 7.8358140 & -4.4842470 \\
\hline 6.0923580 & 5.5307570 & -4.8164230 \\
\hline-0.2723320 & -3.2537690 & 1.1028770 \\
\hline 0.0010910 & -4.0665300 & -0.0196890 \\
\hline 1.2990990 & -4.1327990 & -0.5461750 \\
\hline-0.9426220 & -4.8390390 & -0.6384360 \\
\hline 4.0283840 & -4.8160080 & -0.7668720 \\
\hline 4.1956530 & -5.2657840 & 0.2186900 \\
\hline 3.0004390 & -5.6411180 & -1.5929710 \\
\hline 2.8524970 & -6.6180210 & -1.1207070 \\
\hline 1.6648550 & -4.9151450 & -1.7829530 \\
\hline 1.7933940 & -4.2199260 & -2.6233140 \\
\hline 0.8751030 & -5.6152680 & -2.0558070 \\
\hline 5.2386800 & -4.8234660 & -1.6512590 \\
\hline 2.7076000 & -3.7050270 & 3.5826280 \\
\hline-2.5268730 & -2.0886460 & 1.1397330 \\
\hline-2.4890490 & -0.7764840 & 1.6278900 \\
\hline-1.8481830 & -0.5656610 & 2.4773620 \\
\hline-3.3776570 & -2.3097890 & 0.0516240 \\
\hline-1.8384750 & -4.4552670 & -0.5723610 \\
\hline-3.4520830 & -3.5774480 & -0.5226150 \\
\hline-1.6764260 & -3.2217510 & 1.7204490 \\
\hline-2.1592320 & -4.1556420 & 1.4223690 \\
\hline-1.6408760 & -3.2341190 & 3.2684160 \\
\hline-1.2155940 & -2.2966470 & 3.6455480 \\
\hline-0.9470010 & -4.0257460 & 3.5820030 \\
\hline-3.0004390 & -3.4600600 & 3.9625320 \\
\hline-3.2537690 & 0.2723320 & 1.1028770 \\
\hline-4.0665300 & -0.0010910 & -0.0196890 \\
\hline-4.1327990 & -1.2990990 & -0.5461750 \\
\hline-4.8390390 & 0.9426220 & -0.6384360 \\
\hline-4.4552670 & 1.8384750 & -0.5723610 \\
\hline-3.7050270 & -2.7076000 & 3.5826280 \\
\hline-3.7758480 & -5.0080570 & 2.6044090 \\
\hline-3.5892580 & -4.8504580 & 3.6716270 \\
\hline-4.5433340 & -4.9855950 & 4.1933050 \\
\hline-2.9091240 & -5.6420770 & 4.0117710 \\
\hline-2.8585800 & -3.2477290 & 5.4785230 \\
\hline-2.1559850 & -3.9707270 & 5.9125690 \\
\hline-3.8203370 & -3.3730250 & 5.9884570 \\
\hline-2.4870520 & -2.2426930 & 5.7090740 \\
\hline-4.8160080 & -4.0283840 & -0.7668720 \\
\hline-5.2657840 & -4.1956530 & 0.2186900 \\
\hline-5.6411180 & -3.0004390 & -1.5929710 \\
\hline-6.6180210 & -2.8524970 & -1.1207070 \\
\hline-4.9151450 & -1.6648550 & -1.7829530 \\
\hline-4.2199260 & -1.7933940 & -2.6233140 \\
\hline-5.6152680 & -0.8751030 & -2.0558070 \\
\hline-5.8984110 & -3.6107660 & -2.8914080 \\
\hline-5.4543840 & -4.8996140 & -2.8507820 \\
\hline-4.8234660 & -5.2386800 & -1.6512590 \\
\hline-4.3188250 & -6.5231540 & -1.4613960 \\
\hline-3.8204370 & -6.7923870 & -0.5345100 \\
\hline-4.4534420 & -7.4585420 & -2.4935950 \\
\hline-5.6009650 & -5.8138630 & -3.8919810 \\
\hline-4.0648580 & -8.4639630 & -2.3676940 \\
\hline-5.0877770 & -7.0995840 & -3.6915710 \\
\hline-5.1862040 & -7.8358140 & -4.4842470 \\
\hline-6.0923580 & -5.5307570 & -4.8164230 \\
\hline 3.6107660 & -5.8984110 & -2.8914080 \\
\hline 4.8996140 & -5.4543840 & -2.8507820 \\
\hline 6.5231540 & -4.3188250 & -1.4613960 \\
\hline 6.7923870 & -3.8204370 & -0.5345100 \\
\hline 7.4585420 & -4.4534420 & -2.4935950 \\
\hline 8.4639630 & -4.0648580 & -2.3676940 \\
\hline 7.0995840 & -5.0877770 & -3.6915710 \\
\hline 7.8358140 & -5.1862040 & -4.4842470 \\
\hline 5.8138630 & -5.6009650 & -3.8919810 \\
\hline
\end{tabular}




$\begin{array}{llllllll}\mathrm{H} & 5.5307570 & -6.0923580 & -4.8164230 & \mathrm{C} & 3.2477290 & -2.8585800 & 5.4785230 \\ \mathrm{C} & 4.8504580 & -3.5892580 & 3.6716270 & \mathrm{H} & 3.9707270 & -2.1559850 & 5.9125690 \\ \mathrm{H} & 5.0080570 & -3.7758480 & 2.6044090 & \mathrm{H} & 3.3730250 & -3.8203370 & 5.9884570 \\ \mathrm{H} & 4.9855950 & -4.5433340 & 4.1933050 & \mathrm{H} & 2.2426930 & -2.4870520 & 5.7090740 \\ \mathrm{H} & 5.6420770 & -2.9091240 & 4.0117710 & & & \end{array}$

Benzofuran resorcinarene derivative out-endo (all-S,S, all- $R$ )-4

- DFT B3LYP/6-311+G(d,p)

Total Energy $=-3999.66004543$ a.u.

Cartesian coordinates

\begin{tabular}{|c|c|c|c|}
\hline & $X Y$ & Z & \\
\hline C & -0.5361000 & 3.2495990 & 0.1850990 \\
\hline C & 0.5776880 & 2.5544210 & 0.6700340 \\
\hline $\mathrm{H}$ & 0.4350050 & 1.8972650 & 1.5201040 \\
\hline C & 1.8691590 & 2.6744850 & 0.1436820 \\
\hline C & 2.0355550 & 3.5109520 & -0.9792490 \\
\hline C & 0.9516560 & 4.2287880 & -1.5046740 \\
\hline C & -0.3023850 & 4.0985570 & -0.9063440 \\
\hline 0 & 3.2362030 & 3.6862230 & -1.6119620 \\
\hline $\mathrm{H}$ & 3.8144430 & 2.9058940 & -1.4995880 \\
\hline $\mathrm{O}$ & -1.3890550 & 4.7561010 & -1.4789900 \\
\hline C & -1.2114810 & 6.0783270 & -2.0695580 \\
\hline $\mathrm{H}$ & -1.9369110 & 6.0864930 & -2.8858370 \\
\hline C & 0.2237300 & 6.3562980 & -2.5820860 \\
\hline C & 1.0799150 & 5.1077730 & -2.7218030 \\
\hline $\mathrm{H}$ & 0.7458520 & 4.5664490 & -3.6194190 \\
\hline $\mathrm{H}$ & 2.1202450 & 5.3919210 & -2.8890610 \\
\hline 0 & 0.8357320 & 7.2669290 & -1.6216070 \\
\hline C & -0.1636970 & 7.7989270 & -0.8610800 \\
\hline C & -1.4033400 & 7.1912300 & -1.0744970 \\
\hline C & -2.5327160 & 7.6409580 & -0.3945660 \\
\hline $\mathrm{H}$ & -3.5013450 & 7.1768280 & -0.5597200 \\
\hline C & -2.3955950 & 8.7020150 & 0.5079500 \\
\hline $\mathrm{H}$ & -3.2628040 & 9.0720190 & 1.0455160 \\
\hline C & -1.1396890 & 9.2888490 & 0.7214340 \\
\hline $\mathrm{H}$ & -1.0482490 & 10.1105840 & 1.4260820 \\
\hline C & 0.0003200 & 8.8487170 & 0.0402500 \\
\hline $\mathrm{H}$ & 0.9722160 & 9.3045400 & 0.1950060 \\
\hline C & -1.9406690 & 3.0639820 & 0.7728250 \\
\hline $\mathrm{H}$ & -2.5129870 & 3.9559680 & 0.5034320 \\
\hline C & -1.9539210 & 2.9941170 & 2.3212930 \\
\hline $\mathrm{H}$ & -1.3199000 & 2.1690110 & 2.6652060 \\
\hline $\mathrm{H}$ & -2.9736360 & 2.7376910 & 2.6376480 \\
\hline C & -1.5164910 & 4.2805720 & 3.0523920 \\
\hline $\mathrm{H}$ & -0.5758690 & 4.6272730 & 2.6030580 \\
\hline C & -2.5544210 & 5.4060990 & 2.9190980 \\
\hline $\mathrm{H}$ & -2.7395640 & 5.6794080 & 1.8764150 \\
\hline $\mathrm{H}$ & -2.2159920 & 6.3118670 & 3.4342500 \\
\hline $\mathrm{H}$ & -3.5111550 & 5.1044150 & 3.3653400 \\
\hline C & -1.2459070 & 3.9796480 & 4.5356630 \\
\hline $\mathrm{H}$ & -2.1514430 & 3.6073160 & 5.0323380 \\
\hline $\mathrm{H}$ & -0.9226400 & 4.8804470 & 5.0695550 \\
\hline $\mathrm{H}$ & -0.4639200 & 3.2209970 & 4.6555240 \\
\hline C & 3.0639820 & 1.9406690 & 0.7728250 \\
\hline C & 3.2495990 & 0.5361000 & 0.1850990 \\
\hline $\mathrm{H}$ & 3.9559680 & 2.5129870 & 0.5034320 \\
\hline C & 2.9941170 & 1.9539210 & 2.3212930 \\
\hline C & 2.5544210 & -0.5776880 & 0.6700340 \\
\hline $\mathrm{H}$ & 1.8972650 & -0.4350050 & 1.5201040 \\
\hline C & 4.0985570 & 0.3023850 & -0.9063440 \\
\hline $\mathrm{H}$ & 2.1690110 & 1.3199000 & 2.6652060 \\
\hline $\mathrm{H}$ & 2.7376910 & 2.9736360 & 2.6376480 \\
\hline C & 4.2805720 & 1.5164910 & 3.0523920 \\
\hline $\mathrm{H}$ & 4.6272730 & 0.5758690 & 2.6030580 \\
\hline C & 5.4060990 & 2.5544210 & 2.9190980 \\
\hline $\mathrm{H}$ & 5.6794080 & 2.7395640 & 1.8764150 \\
\hline $\mathrm{H}$ & 6.3118670 & 2.2159920 & 3.4342500 \\
\hline $\mathrm{H}$ & 5.1044150 & 3.5111550 & 3.3653400 \\
\hline C & 3.9796480 & 1.2459070 & 4.5356630 \\
\hline $\mathrm{H}$ & 3.6073160 & 2.1514430 & 5.0323380 \\
\hline $\mathrm{H}$ & 4.8804470 & 0.9226400 & 5.0695550 \\
\hline $\mathrm{H}$ & 3.2209970 & 0.4639200 & 4.6555240 \\
\hline C & 2.6744850 & -1.8691590 & 0.1436820 \\
\hline C & 3.5109520 & -2.0355550 & -0.9792490 \\
\hline C & 4.2287880 & -0.9516560 & -1.5046740 \\
\hline 0 & 3.6862230 & -3.2362030 & -1.6119620 \\
\hline $\mathrm{O}$ & 4.7561010 & 1.3890550 & -1.4789900 \\
\hline c & 6.0783270 & 1.2114810 & -2.0695580 \\
\hline $\mathrm{H}$ & 6.0864930 & 1.9369110 & -2.8858370 \\
\hline C & 6.3562980 & -0.2237300 & -2.5820860 \\
\hline
\end{tabular}

\begin{tabular}{|c|c|c|c|}
\hline C & 5.1077730 & -1.0799150 & -2.7218030 \\
\hline $\mathrm{H}$ & 4.5664490 & -0.7458520 & -3.6194190 \\
\hline $\mathrm{H}$ & 5.3919210 & -2.1202450 & -2.8890610 \\
\hline C & 7.1912300 & 1.4033400 & -1.0744970 \\
\hline C & 0.5361000 & -3.2495990 & 0.1850990 \\
\hline C & -0.5776880 & -2.5544210 & 0.6700340 \\
\hline $\mathrm{H}$ & -0.4350050 & -1.8972650 & 1.5201040 \\
\hline C & 0.3023850 & -4.0985570 & -0.9063440 \\
\hline $\mathrm{H}$ & 2.9058940 & -3.8144430 & -1.4995880 \\
\hline $\mathrm{O}$ & 1.3890550 & -4.7561010 & -1.4789900 \\
\hline C & 1.9406690 & -3.0639820 & 0.7728250 \\
\hline $\mathrm{H}$ & 2.5129870 & -3.9559680 & 0.5034320 \\
\hline C & 1.9539210 & -2.9941170 & 2.3212930 \\
\hline $\mathrm{H}$ & 1.3199000 & -2.1690110 & 2.6652060 \\
\hline $\mathrm{H}$ & 2.9736360 & -2.7376910 & 2.6376480 \\
\hline C & 1.5164910 & -4.2805720 & 3.0523920 \\
\hline 0 & 7.2669290 & -0.8357320 & -1.6216070 \\
\hline C & 7.7989270 & 0.1636970 & -0.8610800 \\
\hline C & 7.6409580 & 2.5327160 & -0.3945660 \\
\hline c & 8.8487170 & -0.0003200 & 0.0402500 \\
\hline $\mathrm{H}$ & 7.1768280 & 3.5013450 & -0.5597200 \\
\hline C & 8.7020150 & 2.3955950 & 0.5079500 \\
\hline $\mathrm{H}$ & 9.0720190 & 3.2628040 & 1.0455160 \\
\hline C & 9.2888490 & 1.1396890 & 0.7214340 \\
\hline $\mathrm{H}$ & 10.1105840 & 1.0482490 & 1.4260820 \\
\hline $\mathrm{H}$ & 9.3045400 & -0.9722160 & 0.1950060 \\
\hline C & -1.8691590 & -2.6744850 & 0.1436820 \\
\hline C & -2.0355550 & -3.5109520 & -0.9792490 \\
\hline C & -0.9516560 & -4.2287880 & -1.5046740 \\
\hline 0 & -3.2362030 & -3.6862230 & -1.6119620 \\
\hline C & 1.2114810 & -6.0783270 & -2.0695580 \\
\hline $\mathrm{H}$ & 1.9369110 & -6.0864930 & -2.8858370 \\
\hline C & -0.2237300 & -6.3562980 & -2.5820860 \\
\hline C & -1.0799150 & -5.1077730 & -2.7218030 \\
\hline $\mathrm{H}$ & -0.7458520 & -4.5664490 & -3.6194190 \\
\hline $\mathrm{H}$ & -2.1202450 & -5.3919210 & -2.8890610 \\
\hline C & 1.4033400 & -7.1912300 & -1.0744970 \\
\hline $\mathrm{H}$ & 0.5758690 & -4.6272730 & 2.6030580 \\
\hline C & -3.2495990 & -0.5361000 & 0.1850990 \\
\hline C & -2.5544210 & 0.5776880 & 0.6700340 \\
\hline $\mathrm{H}$ & -1.8972650 & 0.4350050 & 1.5201040 \\
\hline C & -4.0985570 & -0.3023850 & -0.9063440 \\
\hline $\mathrm{H}$ & -3.8144430 & -2.9058940 & -1.4995880 \\
\hline 0 & -4.7561010 & -1.3890550 & -1.4789900 \\
\hline C & -3.0639820 & -1.9406690 & 0.7728250 \\
\hline $\mathrm{H}$ & -3.9559680 & -2.5129870 & 0.5034320 \\
\hline C & -2.9941170 & -1.9539210 & 2.3212930 \\
\hline $\mathrm{H}$ & -2.1690110 & -1.3199000 & 2.6652060 \\
\hline $\mathrm{H}$ & -2.7376910 & -2.9736360 & 2.6376480 \\
\hline C & -4.2805720 & -1.5164910 & 3.0523920 \\
\hline C & -2.6744850 & 1.8691590 & 0.1436820 \\
\hline C & -3.5109520 & 2.0355550 & -0.9792490 \\
\hline C & -4.2287880 & 0.9516560 & -1.5046740 \\
\hline 0 & -3.6862230 & 3.2362030 & -1.6119620 \\
\hline $\mathrm{H}$ & -2.9058940 & 3.8144430 & -1.4995880 \\
\hline $\mathrm{H}$ & -4.6272730 & -0.5758690 & 2.6030580 \\
\hline $\mathrm{H}$ & -5.6794080 & -2.7395640 & 1.8764150 \\
\hline C & -5.4060990 & -2.5544210 & 2.9190980 \\
\hline $\mathrm{H}$ & -6.3118670 & -2.2159920 & 3.4342500 \\
\hline $\mathrm{H}$ & -5.1044150 & -3.5111550 & 3.3653400 \\
\hline C & -3.9796480 & -1.2459070 & 4.5356630 \\
\hline $\mathrm{H}$ & -3.6073160 & -2.1514430 & 5.0323380 \\
\hline $\mathrm{H}$ & -4.8804470 & -0.9226400 & 5.0695550 \\
\hline $\mathrm{H}$ & -3.2209970 & -0.4639200 & 4.6555240 \\
\hline C & -6.0783270 & -1.2114810 & -2.0695580 \\
\hline $\mathrm{H}$ & -6.0864930 & -1.9369110 & -2.8858370 \\
\hline C & -6.3562980 & 0.2237300 & -2.5820860 \\
\hline C & -5.1077730 & 1.0799150 & -2.7218030 \\
\hline $\mathrm{H}$ & -4.5664490 & 0.7458520 & -3.6194190 \\
\hline $\mathrm{H}$ & -5.3919210 & 2.1202450 & -2.8890610 \\
\hline
\end{tabular}




$\begin{array}{llll}\text { O } & -7.2669290 & 0.8357320 & -1.6216070 \\ \mathrm{C} & -7.7989270 & -0.1636970 & -0.8610800 \\ \mathrm{C} & -7.1912300 & -1.4033400 & -1.0744970 \\ \mathrm{C} & -7.6409580 & -2.5327160 & -0.3945660 \\ \mathrm{H} & -7.1768280 & -3.5013450 & -0.5597200 \\ \mathrm{C} & -8.7020150 & -2.3955950 & 0.5079500 \\ \mathrm{C} & -8.8487170 & 0.0003200 & 0.0402500 \\ \mathrm{H} & -9.0720190 & -3.2628040 & 1.0455160 \\ \mathrm{C} & -9.2888490 & -1.1396890 & 0.7214340 \\ \mathrm{H} & -10.110584 & -1.0482490 & 1.4260820 \\ \mathrm{H} & -9.3045400 & 0.9722160 & 0.1950060 \\ \mathrm{O} & -0.8357320 & -7.2669290 & -1.6216070 \\ \mathrm{C} & 0.1636970 & -7.7989270 & -0.8610800 \\ \mathrm{C} & 2.5327160 & -7.6409580 & -0.3945660 \\ \mathrm{H} & 3.5013450 & -7.1768280 & -0.5597200 \\ \mathrm{C} & 2.3955950 & -8.7020150 & 0.5079500 \\ \mathrm{H} & 3.2628040 & -9.0720190 & 1.0455160\end{array}$

$\begin{array}{llll}\mathrm{C} & 1.1396890 & -9.2888490 & 0.7214340 \\ \mathrm{H} & 1.0482490 & -10.110584 & 1.4260820 \\ \mathrm{C} & -0.0003200 & -8.8487170 & 0.0402500 \\ \mathrm{H} & -0.9722160 & -9.3045400 & 0.1950060 \\ \mathrm{C} & 2.5544210 & -5.4060990 & 2.9190980 \\ \mathrm{H} & 2.7395640 & -5.6794080 & 1.8764150 \\ \mathrm{H} & 2.2159920 & -6.3118670 & 3.4342500 \\ \mathrm{H} & 3.5111550 & -5.1044150 & 3.3653400 \\ \mathrm{C} & 1.2459070 & -3.9796480 & 4.5356630 \\ \mathrm{H} & 2.1514430 & -3.6073160 & 5.0323380 \\ \mathrm{H} & 0.9226400 & -4.8804470 & 5.0695550 \\ \mathrm{H} & 0.4639200 & -3.2209970 & 4.6555240 \\ \mathrm{H} & 0.1654160 & 6.9030880 & -3.5305630 \\ \mathrm{H} & 6.9030880 & -0.1654160 & -3.5305630 \\ \mathrm{H} & -0.1654160 & -6.9030880 & -3.5305630 \\ \mathrm{H} & -6.9030880 & 0.1654160 & -3.5305630\end{array}$

Benzofuran resorcinarene derivative in-exo (all-S,S, all- $R$ )-4 - $\quad$ DFT B3LYP/6-311+G(d,p)

Total Energy = -3999.65513043 a.u.

Cartesian coordinates

\begin{tabular}{|c|c|c|c|}
\hline & $X Y$ & Z & \\
\hline C & -2.8743450 & 1.5694150 & 1.2930840 \\
\hline C & -1.6502960 & 2.0228370 & 1.7983100 \\
\hline $\mathrm{H}$ & -1.2167900 & 1.4908110 & 2.6380560 \\
\hline C & -0.9584310 & 3.1273650 & 1.2905120 \\
\hline C & -1.5380610 & 3.8143380 & 0.2066640 \\
\hline C & -2.7767210 & 3.4190760 & -0.3181270 \\
\hline C & -3.4041020 & 2.2866390 & 0.2101660 \\
\hline $\mathrm{O}$ & -0.9530690 & 4.9164560 & -0.3613380 \\
\hline $\mathrm{H}$ & -0.0005850 & 4.9394820 & -0.1499450 \\
\hline $\mathrm{O}$ & -4.6222960 & 1.8987060 & -0.3484100 \\
\hline C & -4.6544880 & 1.9500660 & -1.8125430 \\
\hline $\mathrm{H}$ & -4.0091910 & 1.1355990 & -2.1591700 \\
\hline C & -4.1871700 & 3.3096190 & -2.3934960 \\
\hline C & -3.5127050 & 4.2168830 & -1.3639600 \\
\hline $\mathrm{H}$ & -2.8491640 & 4.9326050 & -1.8502150 \\
\hline $\mathrm{O}$ & -5.3681320 & 3.9738100 & -2.9271540 \\
\hline C & -6.3939970 & 3.0740700 & -2.9196820 \\
\hline C & -6.0694500 & 1.8595970 & -2.3094140 \\
\hline C & -7.0135160 & 0.8354320 & -2.2439570 \\
\hline $\mathrm{H}$ & -6.7691740 & -0.1161000 & -1.7823520 \\
\hline C & -8.2843120 & 1.0573730 & -2.7872260 \\
\hline $\mathrm{H}$ & -9.0329110 & 0.2725470 & -2.7469900 \\
\hline C & -8.5938320 & 2.2866430 & -3.3861520 \\
\hline $\mathrm{H}$ & -9.5841490 & 2.4430480 & -3.8043720 \\
\hline C & -7.6520460 & 3.3176190 & -3.4658880 \\
\hline $\mathrm{H}$ & -7.8810290 & 4.2688770 & -3.9337950 \\
\hline C & -3.6064890 & 0.3661030 & 1.8954490 \\
\hline $\mathrm{H}$ & -4.6598610 & 0.4737840 & 1.6257120 \\
\hline C & -3.5890830 & 0.3461890 & 3.4445820 \\
\hline $\mathrm{H}$ & -2.5577780 & 0.2907140 & 3.8118650 \\
\hline $\mathrm{H}$ & -4.0734140 & -0.5840900 & 3.7703450 \\
\hline C & -4.2868990 & 1.5380610 & 4.1336310 \\
\hline $\mathrm{H}$ & -3.8374280 & 2.4642460 & 3.7504080 \\
\hline C & -5.7961610 & 1.5892480 & 3.8441060 \\
\hline $\mathrm{H}$ & -6.0140660 & 1.7229090 & 2.7798090 \\
\hline $\mathrm{H}$ & -6.2631700 & 2.4248460 & 4.3770600 \\
\hline $\mathrm{H}$ & -6.2891960 & 0.6664550 & 4.1764960 \\
\hline C & -4.0343090 & 1.4854490 & 5.6492960 \\
\hline $\mathrm{H}$ & -4.4576060 & 0.5722620 & 6.0870830 \\
\hline $\mathrm{H}$ & -4.4954520 & 2.3401950 & 6.1563980 \\
\hline $\mathrm{H}$ & -2.9626130 & 1.4992910 & 5.8792640 \\
\hline C & 0.3661030 & 3.6064890 & 1.8954490 \\
\hline C & 1.5694150 & 2.8743450 & 1.2930840 \\
\hline $\mathrm{H}$ & 0.4737840 & 4.6598610 & 1.6257120 \\
\hline C & 0.3461890 & 3.5890830 & 3.4445820 \\
\hline C & 2.0228370 & 1.6502960 & 1.7983100 \\
\hline $\mathrm{H}$ & 1.4908110 & 1.2167900 & 2.6380560 \\
\hline C & 2.2866390 & 3.4041020 & 0.2101660 \\
\hline $\mathrm{H}$ & 0.2907140 & 2.5577780 & 3.8118650 \\
\hline $\mathrm{H}$ & -0.5840900 & 4.0734140 & 3.7703450 \\
\hline C & 1.5380610 & 4.2868990 & 4.1336310 \\
\hline $\mathrm{H}$ & 2.4642460 & 3.8374280 & 3.7504080 \\
\hline C & 1.5892480 & 5.7961610 & 3.8441060 \\
\hline $\mathrm{H}$ & 1.7229090 & 6.0140660 & 2.7798090 \\
\hline $\mathrm{H}$ & 2.4248460 & 6.2631700 & 4.3770600 \\
\hline $\mathrm{H}$ & 0.6664550 & 6.2891960 & 4.1764960 \\
\hline C & 1.4854490 & 4.0343090 & 5.6492960 \\
\hline
\end{tabular}

\begin{tabular}{|c|c|c|}
\hline 0.5722620 & 4.4576060 & 6.0870830 \\
\hline 2.3401950 & 4.4954520 & 6.1563980 \\
\hline 1.4992910 & 2.9626130 & 5.8792640 \\
\hline 3.1273650 & 0.9584310 & 1.2905120 \\
\hline 3.8143380 & 1.5380610 & 0.2066640 \\
\hline 3.4190760 & 2.7767210 & -0.3181270 \\
\hline 4.9164560 & 0.9530690 & -0.3613380 \\
\hline 1.8987060 & 4.6222960 & -0.3484100 \\
\hline 1.9500660 & 4.6544880 & -1.8125430 \\
\hline 1.1355990 & 4.0091910 & -2.1591700 \\
\hline 3.3096190 & 4.1871700 & -2.3934960 \\
\hline 4.2168830 & 3.5127050 & -1.3639600 \\
\hline 4.9326050 & 2.8491640 & -1.8502150 \\
\hline 1.8595970 & 6.0694500 & -2.3094140 \\
\hline 2.8743450 & -1.5694150 & 1.2930840 \\
\hline 1.6502960 & -2.0228370 & 1.7983100 \\
\hline 1.2167900 & -1.4908110 & 2.6380560 \\
\hline 3.4041020 & -2.2866390 & 0.2101660 \\
\hline 4.9394820 & 0.0005850 & -0.1499450 \\
\hline 4.6222960 & -1.8987060 & -0.3484100 \\
\hline 3.6064890 & -0.3661030 & 1.8954490 \\
\hline 4.6598610 & -0.4737840 & 1.6257120 \\
\hline 3.5890830 & -0.3461890 & 3.4445820 \\
\hline 2.5577780 & -0.2907140 & 3.8118650 \\
\hline 4.0734140 & 0.5840900 & 3.7703450 \\
\hline 4.2868990 & -1.5380610 & 4.1336310 \\
\hline 3.9738100 & 5.3681320 & -2.9271540 \\
\hline 3.0740700 & 6.3939970 & -2.9196820 \\
\hline 0.8354320 & 7.0135160 & -2.2439570 \\
\hline 3.3176190 & 7.6520460 & -3.4658880 \\
\hline-0.1161000 & 6.7691740 & -1.7823520 \\
\hline 1.0573730 & 8.2843120 & -2.7872260 \\
\hline 0.2725470 & 9.0329110 & -2.7469900 \\
\hline 2.2866430 & 8.5938320 & -3.3861520 \\
\hline 2.4430480 & 9.5841490 & -3.8043720 \\
\hline 4.2688770 & 7.8810290 & -3.9337950 \\
\hline 0.9584310 & -3.1273650 & 1.2905120 \\
\hline 1.5380610 & -3.8143380 & 0.2066640 \\
\hline 2.7767210 & -3.4190760 & -0.3181270 \\
\hline 0.9530690 & -4.9164560 & -0.3613380 \\
\hline 4.6544880 & -1.9500660 & -1.8125430 \\
\hline 4.0091910 & -1.1355990 & -2.1591700 \\
\hline 4.1871700 & -3.3096190 & -2.3934960 \\
\hline 3.5127050 & -4.2168830 & -1.3639600 \\
\hline 2.8491640 & -4.9326050 & -1.8502150 \\
\hline 6.0694500 & -1.8595970 & -2.3094140 \\
\hline 3.8374280 & -2.4642460 & 3.7504080 \\
\hline-1.5694150 & -2.8743450 & 1.2930840 \\
\hline-2.0228370 & -1.6502960 & 1.7983100 \\
\hline-1.4908110 & -1.2167900 & 2.6380560 \\
\hline-2.2866390 & -3.4041020 & 0.2101660 \\
\hline 0.0005850 & -4.9394820 & -0.1499450 \\
\hline-1.8987060 & -4.6222960 & -0.3484100 \\
\hline-0.3661030 & -3.6064890 & 1.8954490 \\
\hline-0.4737840 & -4.6598610 & 1.6257120 \\
\hline-0.3461890 & -3.5890830 & 3.4445820 \\
\hline-0.2907140 & -2.5577780 & 3.8118650 \\
\hline 0.5840900 & -4.0734140 & 3.7703450 \\
\hline
\end{tabular}




$\begin{array}{lll}-1.5380610 & -4.2868990 & 4.1336310 \\ -3.1273650 & -0.9584310 & 1.2905120 \\ -3.8143380 & -1.5380610 & 0.2066640 \\ -3.4190760 & -2.7767210 & -0.3181270 \\ -4.9164560 & -0.9530690 & -0.3613380 \\ -4.9394820 & -0.0005850 & -0.1499450 \\ -2.4642460 & -3.8374280 & 3.7504080 \\ -1.7229090 & -6.0140660 & 2.7798090 \\ -1.5892480 & -5.7961610 & 3.8441060 \\ -2.4248460 & -6.2631700 & 4.3770600 \\ -0.6664550 & -6.2891960 & 4.1764960 \\ -1.4854490 & -4.0343090 & 5.6492960 \\ -0.5722620 & -4.4576060 & 6.0870830 \\ -2.3401950 & -4.4954520 & 6.1563980 \\ -1.4992910 & -2.9626130 & 5.8792640 \\ -1.9500660 & -4.6544880 & -1.8125430 \\ -1.1355990 & -4.0091910 & -2.1591700 \\ -3.3096190 & -4.1871700 & -2.3934960 \\ -4.2168830 & -3.5127050 & -1.3639600 \\ -4.9326050 & -2.8491640 & -1.8502150 \\ -3.9738100 & -5.3681320 & -2.9271540 \\ -3.0740700 & -6.3939970 & -2.9196820 \\ -1.8595970 & -6.0694500 & -2.3094140 \\ -0.8354320 & -7.0135160 & -2.2439570 \\ 0.1161000 & -6.7691740 & -1.7823520 \\ -1.0573730 & -8.2843120 & -2.7872260 \\ -3.3176190 & -7.6520460 & -3.4658880 \\ -0.2725470 & -9.0329110 & -2.7469900 \\ -2.2866430 & -8.5938320 & -3.3861520\end{array}$

$\begin{array}{llll}H & -2.4430480 & -9.5841490 & -3.8043720 \\ H & -4.2688770 & -7.8810290 & -3.9337950 \\ \text { O } & 5.3681320 & -3.9738100 & -2.9271540 \\ \mathrm{C} & 6.3939970 & -3.0740700 & -2.9196820 \\ \mathrm{C} & 7.0135160 & -0.8354320 & -2.2439570 \\ \mathrm{H} & 6.7691740 & 0.1161000 & -1.7823520 \\ \mathrm{C} & 8.2843120 & -1.0573730 & -2.7872260 \\ \mathrm{H} & 9.0329110 & -0.2725470 & -2.7469900 \\ \mathrm{C} & 8.5938320 & -2.2866430 & -3.3861520 \\ \mathrm{H} & 9.5841490 & -2.4430480 & -3.8043720 \\ \mathrm{C} & 7.6520460 & -3.3176190 & -3.4658880 \\ \mathrm{H} & 7.8810290 & -4.2688770 & -3.9337950 \\ \mathrm{C} & 5.7961610 & -1.5892480 & 3.8441060 \\ \mathrm{H} & 6.0140660 & -1.7229090 & 2.7798090 \\ \mathrm{H} & 6.2631700 & -2.4248460 & 4.3770600 \\ \mathrm{H} & 6.2891960 & -0.6664550 & 4.1764960 \\ \mathrm{C} & 4.0343090 & -1.4854490 & 5.6492960 \\ \mathrm{H} & 4.4576060 & -0.5722620 & 6.0870830 \\ \mathrm{H} & 4.4954520 & -2.3401950 & 6.1563980 \\ \mathrm{H} & 2.9626130 & -1.4992910 & 5.8792640 \\ \mathrm{H} & -3.5255310 & 3.1206840 & -3.2462300 \\ \mathrm{H} & 3.1206840 & 3.5255310 & -3.2462300 \\ \mathrm{H} & 3.5255310 & -3.1206840 & -3.2462300 \\ \mathrm{H} & -3.1206840 & -3.5255310 & -3.2462300 \\ \mathrm{H} & 4.7935760 & 4.3098750 & -0.8763250 \\ \mathrm{H} & -4.3098750 & 4.7935760 & -0.8763250 \\ \mathrm{H} & -4.7935760 & -4.3098750 & -0.8763250 \\ \mathrm{H} & 4.3098750 & -4.7935760 & -0.8763250\end{array}$

Benzofuran resorcinarene derivative out-endo (all- $R, R$, all- $R$ )-5 - $\quad$ DFT B3LYP/6-311+G(d,p)

Total Energy $=-3999.68486288$ a.u.

Cartesian coordinates

\begin{tabular}{|c|c|c|c|}
\hline & $x$ & $Y$ & Z \\
\hline C & 3.0090670 & 1.3127070 & 0.1451240 \\
\hline C & 2.6067630 & 0.0613170 & 0.6287990 \\
\hline $\mathrm{H}$ & 1.9277170 & 0.0365190 & 1.4739060 \\
\hline C & 3.0525720 & -1.1584310 & 0.1063530 \\
\hline C & 3.9181830 & -1.1164640 & -1.0083170 \\
\hline C & 4.3509200 & 0.1115780 & -1.5261730 \\
\hline C & 3.8973980 & 1.2880910 & -0.9332790 \\
\hline $\mathrm{O}$ & 4.3996530 & -2.2359900 & -1.6308150 \\
\hline $\mathrm{H}$ & 3.7978670 & -2.9956990 & -1.5157380 \\
\hline 0 & 4.2875310 & 2.4983360 & -1.5200370 \\
\hline C & 5.6500320 & 2.6584670 & -1.9006910 \\
\hline C & 6.3152110 & 1.3578720 & -2.4456950 \\
\hline $\mathrm{H}$ & 6.8239800 & 1.5987640 & -3.3873450 \\
\hline C & 5.2791090 & 0.2454330 & -2.7047770 \\
\hline $\mathrm{H}$ & 4.7043090 & 0.4980100 & -3.6071620 \\
\hline $\mathrm{H}$ & 5.7846750 & -0.7008740 & -2.9055780 \\
\hline C & 2.4983360 & 2.6333020 & 0.7283120 \\
\hline $\mathrm{H}$ & 3.2243600 & 3.4001390 & 0.4470680 \\
\hline C & 2.4517050 & 2.6446340 & 2.2765240 \\
\hline $\mathrm{H}$ & 1.7809190 & 1.8578220 & 2.6413550 \\
\hline $\mathrm{H}$ & 1.9977720 & 3.5942940 & 2.5898170 \\
\hline C & 3.8122040 & 2.4863840 & 2.9872310 \\
\hline $\mathrm{H}$ & 4.2898750 & 1.5718220 & 2.6104420 \\
\hline C & 4.7615100 & 3.6646500 & 2.7140070 \\
\hline $\mathrm{H}$ & 5.0263880 & 3.7505910 & 1.6561180 \\
\hline $\mathrm{H}$ & 5.6956720 & 3.5430800 & 3.2742940 \\
\hline $\mathrm{H}$ & 4.3068600 & 4.6128330 & 3.0298410 \\
\hline C & 3.5932960 & 2.3139550 & 4.4993150 \\
\hline $\mathrm{H}$ & 3.1081920 & 3.1998040 & 4.9294210 \\
\hline $\mathrm{H}$ & 4.5455450 & 2.1709430 & 5.0222760 \\
\hline $\mathrm{H}$ & 2.9583700 & 1.4473750 & 4.7175980 \\
\hline C & 2.6333020 & -2.4983360 & 0.7283120 \\
\hline C & 1.3127070 & -3.0090670 & 0.1451240 \\
\hline $\mathrm{H}$ & 3.4001390 & -3.2243600 & 0.4470680 \\
\hline C & 2.6446340 & -2.4517050 & 2.2765240 \\
\hline C & 0.0613170 & -2.6067630 & 0.6287990 \\
\hline $\mathrm{H}$ & 0.0365190 & -1.9277170 & 1.4739060 \\
\hline C & 1.2880910 & -3.8973980 & -0.9332790 \\
\hline $\mathrm{H}$ & 1.8578220 & -1.7809190 & 2.6413550 \\
\hline $\mathrm{H}$ & 3.5942940 & -1.9977720 & 2.5898170 \\
\hline C & 2.4863840 & -3.8122040 & 2.9872310 \\
\hline $\mathrm{H}$ & 1.5718220 & -4.2898750 & 2.6104420 \\
\hline C & 3.6646500 & -4.7615100 & 2.7140070 \\
\hline $\mathrm{H}$ & 3.7505910 & -5.0263880 & 1.6561180 \\
\hline $\mathrm{H}$ & 3.5430800 & -5.6956720 & 3.2742940 \\
\hline
\end{tabular}

\begin{tabular}{llll} 
H & 4.6128330 & -4.3068600 & 3.0298410 \\
$\mathrm{C}$ & 2.3139550 & -3.5932960 & 4.4993150 \\
$\mathrm{H}$ & 3.1998040 & -3.1081920 & 4.9294210 \\
$\mathrm{H}$ & 2.1709430 & -4.5455450 & 5.0222760 \\
$\mathrm{H}$ & 1.4473750 & -2.9583700 & 4.7175980 \\
$\mathrm{C}$ & -1.1584310 & -3.0525720 & 0.1063530 \\
$\mathrm{C}$ & -1.1164640 & -3.9181830 & -1.0083170 \\
$\mathrm{C}$ & 0.1115780 & -4.3509200 & -1.5261730 \\
$\mathrm{O}$ & -2.2359900 & -4.3996530 & -1.6308150 \\
$\mathrm{O}$ & 2.4983360 & -4.2875310 & -1.5200370 \\
$\mathrm{C}$ & 2.6584670 & -5.6500320 & -1.9006910 \\
$\mathrm{C}$ & 1.3578720 & -6.3152110 & -2.4456950 \\
$\mathrm{H}$ & 1.5987640 & -6.8239800 & -3.3873450 \\
$\mathrm{C}$ & -.2454330 & -5.2791090 & -2.7047770 \\
$\mathrm{H}$ & 0.4980100 & -4.7043090 & -3.6071620 \\
$\mathrm{H}$ & -0.7008740 & -5.7846750 & -2.9055780 \\
$\mathrm{C}$ & -3.0090670 & -1.3127070 & 0.1451240 \\
$\mathrm{C}$ & -2.6067630 & -0.0613170 & 0.6287990 \\
$\mathrm{H}$ & -1.9277170 & -0.0365190 & 1.4739060 \\
$\mathrm{C}$ & -3.8973980 & -1.2880910 & -0.9332790 \\
$\mathrm{H}$ & -2.9956990 & -3.7978670 & -1.5157380 \\
$\mathrm{O}$ & -4.2875310 & -2.4983360 & -1.5200370 \\
$\mathrm{C}$ & -2.4983360 & -2.6333020 & 0.7283120 \\
$\mathrm{H}$ & -3.2243600 & -3.4001390 & 0.4470680 \\
$\mathrm{C}$ & -2.4517050 & -2.6446340 & 2.2765240 \\
$\mathrm{H}$ & -1.7809190 & -1.8578220 & 2.6413550 \\
$\mathrm{H}$ & -1.9977720 & -3.5942940 & 2.5898170 \\
$\mathrm{C}$ & -3.8122040 & -2.4863840 & 2.9872310 \\
$\mathrm{C}$ & -3.0525720 & 1.1584310 & 0.1063530 \\
$\mathrm{C}$ & -3.9181830 & 1.1164640 & -1.0083170 \\
$\mathrm{C}$ & -4.3509200 & -0.1115780 & -1.5261730 \\
$\mathrm{O}$ & -4.3996530 & 2.2359900 & -1.6308150 \\
$\mathrm{C}$ & -5.6500320 & -2.6584670 & -1.9006910 \\
$\mathrm{C}$ & -6.3152110 & -1.3578720 & -2.4456950 \\
$\mathrm{H}$ & -6.8239800 & -1.5987640 & -3.3873450 \\
$\mathrm{C}$ & -5.2791090 & -0.2454330 & -2.7047770 \\
$\mathrm{H}$ & -4.7043090 & -0.4980100 & -3.6071620 \\
$\mathrm{H}$ & -5.7846750 & 0.7008740 & -2.9055780 \\
$\mathrm{H}$ & -4.2898750 & -1.5718220 & 2.6104420 \\
$\mathrm{C}$ & -1.3127070 & 3.0090670 & 0.1451240 \\
$\mathrm{C}$ & -0.0613170 & 2.6067630 & 0.6287990 \\
$\mathrm{H}$ & -0.0365190 & 1.9277170 & 1.4739060 \\
$\mathrm{C}$ & -1.2880910 & 3.8973980 & -0.9332790 \\
$\mathrm{O}$ & -3.7978670 & 2.9956990 & -1.5157380 \\
\hline-2.4983360 & 4.2875310 & -1.5200370 \\
\hline & -2.6333020 & 2.4983360 & 0.7283120
\end{tabular}




$\begin{array}{lll}-3.4001390 & 3.2243600 & 0.4470680 \\ -2.6446340 & 2.4517050 & 2.2765240 \\ -1.8578220 & 1.7809190 & 2.6413550 \\ -3.5942940 & 1.9977720 & 2.5898170 \\ -2.4863840 & 3.8122040 & 2.9872310 \\ 1.1584310 & 3.0525720 & 0.1063530 \\ 1.1164640 & 3.9181830 & -1.0083170 \\ -0.1115780 & 4.3509200 & -1.5261730 \\ 2.2359900 & 4.3996530 & -1.6308150 \\ 2.9956990 & 3.7978670 & -1.5157380 \\ -1.5718220 & 4.2898750 & 2.6104420 \\ -3.7505910 & 5.0263880 & 1.6561180 \\ -3.6646500 & 4.7615100 & 2.7140070 \\ -3.5430800 & 5.6956720 & 3.2742940 \\ -4.6128330 & 4.3068600 & 3.0298410 \\ -2.3139550 & 3.5932960 & 4.4993150 \\ -3.1998040 & 3.1081920 & 4.9294210 \\ -2.1709430 & 4.5455450 & 5.0222760 \\ -1.4473750 & 2.9583700 & 4.7175980 \\ -2.6584670 & 5.6500320 & -1.9006910 \\ -1.3578720 & 6.3152110 & -2.4456950 \\ -1.5987640 & 6.8239800 & -3.3873450 \\ -0.2454330 & 5.2791090 & -2.7047770 \\ -0.4980100 & 4.7043090 & -3.6071620 \\ 0.7008740 & 5.7846750 & -2.9055780 \\ -4.7615100 & -3.6646500 & 2.7140070 \\ -5.0263880 & -3.7505910 & 1.6561180 \\ -5.6956720 & -3.5430800 & 3.2742940 \\ -4.3068600 & -4.6128330 & 3.0298410 \\ -3.5932960 & -2.3139550 & 4.4993150 \\ -3.1081920 & -3.1998040 & 4.9294210 \\ -4.5455450 & -2.1709430 & 5.0222760 \\ -2.9583700 & -1.4473750 & 4.7175980 \\ 3.0913360 & -6.4202150 & -0.7691300 \\ 1.0570810 & -7.3333070 & -1.3668900 \\ 2.1072040 & -7.3313870 & -0.4512510 \\ -0.0135390 & -8.2015960 & -1.1825820 \\ 2.1481270 & -8.1750290 & 0.6512690 \\ -0.0034840 & -9.0660120 & -0.0779980 \\ -0.8482920 & -8.2108060 & -1.8781910 \\ 1.0671490 & -9.0503480 & 0.8232140\end{array}$

\begin{tabular}{llll} 
H & 2.9809510 & -8.1497280 & 1.3454820 \\
H & -0.8319440 & -9.7496020 & 0.0790620 \\
H & 1.0622230 & -9.7244960 & 1.6747620 \\
O & -6.4202150 & -3.0913360 & -0.7691300 \\
$\mathrm{C}$ & -7.3333070 & -1.0570810 & -1.3668900 \\
$\mathrm{C}$ & -7.3313870 & -2.1072040 & -0.4512510 \\
$\mathrm{C}$ & -8.2015960 & 0.0135390 & -1.1825820 \\
$\mathrm{C}$ & -8.1750290 & -2.1481270 & 0.6512690 \\
$\mathrm{C}$ & -9.0660120 & 0.0034840 & -0.0779980 \\
$\mathrm{H}$ & -8.2108060 & 0.8482920 & -1.8781910 \\
$\mathrm{C}$ & -9.0503480 & -1.0671490 & 0.8232140 \\
$\mathrm{H}$ & -8.1497280 & -2.9809510 & 1.3454820 \\
$\mathrm{H}$ & -9.7496020 & 0.8319440 & 0.0790620 \\
$\mathrm{H}$ & -9.7244960 & -1.0622230 & 1.6747620 \\
$\mathrm{O}$ & -3.0913360 & 6.4202150 & -0.7691300 \\
$\mathrm{C}$ & -1.0570810 & 7.3333070 & -1.3668900 \\
$\mathrm{C}$ & -2.1072040 & 7.3313870 & -0.4512510 \\
$\mathrm{C}$ & 0.0135390 & 8.2015960 & -1.1825820 \\
$\mathrm{C}$ & -2.1481270 & 8.1750290 & 0.6512690 \\
$\mathrm{C}$ & 0.0034840 & 9.0660120 & -0.0779980 \\
$\mathrm{H}$ & 0.8482920 & 8.2108060 & -1.8781910 \\
$\mathrm{C}$ & -1.0671490 & 9.0503480 & 0.8232140 \\
$\mathrm{H}$ & -2.9809510 & 8.1497280 & 1.3454820 \\
$\mathrm{H}$ & 0.8319440 & 9.7496020 & 0.0790620 \\
$\mathrm{H}$ & -1.0622230 & 9.7244960 & 1.6747620 \\
$\mathrm{O}$ & 6.4202150 & 3.0913360 & -0.7691300 \\
$\mathrm{C}$ & 7.3333070 & 1.0570810 & -1.3668900 \\
$\mathrm{C}$ & 7.3313870 & 2.1072040 & -0.4512510 \\
$\mathrm{C}$ & 8.2015960 & -0.0135390 & -1.1825820 \\
$\mathrm{C}$ & 8.1750290 & 2.1481270 & 0.6512690 \\
$\mathrm{C}$ & 9.0660120 & -0.0034840 & -0.0779980 \\
$\mathrm{H}$ & 8.2108060 & -0.8482920 & -1.8781910 \\
$\mathrm{C}$ & 9.0503480 & 1.0671490 & 0.8232140 \\
$\mathrm{H}$ & 8.1497280 & 2.9809510 & 1.3454820 \\
$\mathrm{H}$ & 9.7496020 & -0.8319440 & 0.0790620 \\
$\mathrm{H}$ & 9.7244960 & 1.0622230 & 1.6747620 \\
$\mathrm{H}$ & -5.6293320 & -3.4838420 & -2.6125410 \\
$\mathrm{H}$ & 3.4838420 & -5.6293320 & -2.6125410 \\
$\mathrm{H}$ & -3.4838420 & 5.6293320 & -2.6125410 \\
$\mathrm{H}$ & 5.6293320 & 3.4838420 & -2.6125410 \\
& & & \\
\hline & & & \\
\hline & & &
\end{tabular}

Benzofuran resorcinarene derivative out-exo (all-S,S, all- $R$ )-5

- $\quad$ DFT B3LYP/6-311+G(d,p)

Total Energy $=-3999.67318572$ a.u.

Cartesian coordinates

\begin{tabular}{llll} 
& $X$ & $Y$ & $Z$ \\
C & -2.0868290 & 2.5254870 & 1.3767090 \\
C & -0.7738780 & 2.4882130 & 1.8621670 \\
H & -0.5600530 & 1.8457450 & 2.7096180 \\
C & 0.2732070 & 3.2561270 & 1.3369910 \\
C & -0.0021040 & 4.0724660 & 0.2169160 \\
C & -1.3015900 & 4.1372830 & -0.3073010 \\
C & -2.3067080 & 3.3773350 & 0.2901670 \\
O & 0.9333300 & 4.8516640 & -0.4038000 \\
H & 1.8392300 & 4.5015790 & -0.3086910 \\
O & -3.5781040 & 3.4513280 & -0.2815770 \\
C & -4.0343960 & 4.7995420 & -0.4917580 \\
H & -4.2298660 & 5.2362120 & 0.4928850 \\
C & -3.0452370 & 5.6317510 & -1.3610850 \\
H & -2.8832480 & 6.5963220 & -0.8643560 \\
C & -1.6766570 & 4.9260610 & -1.5362700 \\
H & -1.7484720 & 4.2365810 & -2.3876610 \\
H & -0.9011720 & 5.6538120 & -1.7783870 \\
C & -3.2220830 & 1.6766570 & 1.9557630 \\
H & -4.1549320 & 2.1624050 & 1.6590110 \\
C & -3.2347930 & 1.6403730 & 3.5038090 \\
H & -2.2939860 & 1.2222300 & 3.8806670 \\
H & -4.0212660 & 0.9408610 & 3.8174830 \\
C & -3.4713480 & 2.9990940 & 4.1961730 \\
H & -2.7324950 & 3.7123460 & 3.8055530 \\
C & -4.8732890 & 3.5671520 & 3.9192960 \\
H & -5.0518320 & 3.7389710 & 2.8529550 \\
H & -5.0126330 & 4.5253020 & 4.4323810 \\
H & -5.6507250 & 2.8811700 & 4.2796120 \\
C & -3.2399520 & 2.8655530 & 5.7101100 \\
H & -3.9462610 & 2.1521110 & 6.1538580 \\
H & -3.3763230 & 3.8265620 & 6.2186120 \\
H & -2.2262910 & 2.5118890 & 5.9308410 \\
C & 1.6766570 & 3.2220830 & 1.9557630 \\
& & & \\
\hline
\end{tabular}

\begin{tabular}{lll}
2.5254870 & 2.0868290 & 1.3767090 \\
2.1624050 & 4.1549320 & 1.6590110 \\
1.6403730 & 3.2347930 & 3.5038090 \\
2.4882130 & 0.7738780 & 1.8621670 \\
1.8457450 & 0.5600530 & 2.7096180 \\
3.3773350 & 2.3067080 & 0.2901670 \\
1.2222300 & 2.2939860 & 3.8806670 \\
0.9408610 & 4.0212660 & 3.8174830 \\
2.9990940 & 3.4713480 & 4.1961730 \\
3.7123460 & 2.7324950 & 3.8055530 \\
3.5671520 & 4.8732890 & 3.9192960 \\
3.7389710 & 5.0518320 & 2.8529550 \\
4.5253020 & 5.0126330 & 4.4323810 \\
2.8811700 & 5.6507250 & 4.2796120 \\
2.8655530 & 3.2399520 & 5.7101100 \\
2.1521110 & 3.9462610 & 6.1538580 \\
3.8265620 & 3.3763230 & 6.2186120 \\
2.5118890 & 2.2262910 & 5.9308410 \\
3.2561270 & -0.2732070 & 1.3369910 \\
4.0724660 & 0.0021040 & 0.2169160 \\
4.1372830 & 1.3015900 & -0.3073010 \\
4.8516640 & -0.933300 & -0.4038000 \\
3.4513280 & 3.5781040 & -0.2815770 \\
4.7995420 & 4.0343960 & -0.4917580 \\
5.2362120 & 4.2298660 & 0.4928850 \\
5.6317510 & 3.0452370 & -1.3610850 \\
6.5963220 & 2.8832480 & -0.8643560 \\
4.9260610 & 1.6766570 & -1.5362700 \\
4.2365810 & 1.7484720 & -2.3876610 \\
5.6538120 & 0.9011720 & -1.7783870 \\
2.0868290 & -2.5254870 & 1.3767090 \\
0.7738780 & -2.4882130 & 1.8621670 \\
0.5600530 & -1.8457450 & 2.7096180 \\
2.3067080 & -3.3773350 & 0.2901670 \\
& & \\
\hline
\end{tabular}




$\begin{array}{lll}4.5015790 & -1.8392300 & -0.3086910 \\ 3.5781040 & -3.4513280 & -0.2815770 \\ 3.2220830 & -1.6766570 & 1.9557630 \\ 4.1549320 & -2.1624050 & 1.6590110 \\ 3.2347930 & -1.6403730 & 3.5038090 \\ 2.2939860 & -1.2222300 & 3.8806670 \\ 4.0212660 & -0.9408610 & 3.8174830 \\ 3.4713480 & -2.9990940 & 4.1961730 \\ -0.2732070 & -3.2561270 & 1.3369910 \\ 0.0021040 & -4.0724660 & 0.2169160 \\ 1.3015900 & -4.1372830 & -0.3073010 \\ -0.9333300 & -4.8516640 & -0.4038000 \\ 4.0343960 & -4.7995420 & -0.4917580 \\ 4.2298660 & -5.2362120 & 0.4928850 \\ 3.0452370 & -5.6317510 & -1.3610850 \\ 2.8832480 & -6.5963220 & -0.8643560 \\ 1.6766570 & -4.9260610 & -1.5362700 \\ 1.7484720 & -4.2365810 & -2.3876610 \\ 0.9011720 & -5.6538120 & -1.7783870 \\ 2.7324950 & -3.7123460 & 3.8055530 \\ -2.5254870 & -2.0868290 & 1.3767090 \\ -2.4882130 & -0.7738780 & 1.8621670 \\ -1.8457450 & -0.5600530 & 2.7096180 \\ -3.3773350 & -2.3067080 & 0.2901670 \\ -1.8392300 & -4.5015790 & -0.3086910 \\ -3.4513280 & -3.5781040 & -0.2815770 \\ -1.6766570 & -3.2220830 & 1.9557630 \\ -2.1624050 & -4.1549320 & 1.6590110 \\ -1.6403730 & -3.2347930 & 3.5038090 \\ -1.2222300 & -2.2939860 & 3.8806670 \\ -0.9408610 & -4.0212660 & 3.8174830 \\ -2.9990940 & -3.4713480 & 4.1961730 \\ -3.2561270 & 0.2732070 & 1.3369910 \\ -4.0724660 & -0.0021040 & 0.2169160 \\ -4.1372830 & -1.3015900 & -0.3073010 \\ -4.8516640 & 0.9333300 & -0.4038000 \\ -4.5015790 & 1.8392300 & -0.3086910 \\ -3.7123460 & -2.7324950 & 3.8055530 \\ -3.7389710 & -5.0518320 & 2.8529550 \\ -3.5671520 & -4.8732890 & 3.9192960 \\ -4.5253020 & -5.0126330 & 4.4323810 \\ -2.8811700 & -5.6507250 & 4.2796120 \\ -2.8655530 & -3.2399520 & 5.7101100 \\ -2.1521110 & -3.9462610 & 6.1538580 \\ -3.8265620 & -3.3763230 & 6.2186120 \\ -2.5118890 & -2.2262910 & 5.9308410 \\ -4.7995420 & -4.0343960 & -0.4917580 \\ -5.2362120 & -4.2298660 & 0.4928850 \\ -5.6317510 & -3.0452370 & -1.3610850 \\ -6.5963220 & -2.8832480 & -0.8643560 \\ -4.9260610 & -1.6766570 & -1.5362700 \\ -5.6538120 & -1.7484720 & -2.3876610 \\ & -0.9011720 & -1.7783870\end{array}$

\begin{tabular}{lll}
4.8732890 & -3.5671520 & 3.9192960 \\
5.0518320 & -3.7389710 & 2.8529550 \\
5.0126330 & -4.5253020 & 4.4323810 \\
5.6507250 & -2.8811700 & 4.2796120 \\
3.2399520 & -2.8655530 & 5.7101100 \\
3.9462610 & -2.1521110 & 6.1538580 \\
3.3763230 & -3.8265620 & 6.2186120 \\
2.2262910 & -2.5118890 & 5.9308410 \\
4.7027800 & 5.2649430 & -1.1836970 \\
5.8072570 & 3.8445870 & -2.6331440 \\
5.2306840 & 5.0999240 & -2.4488320 \\
6.3949470 & 3.5341350 & -3.8555600 \\
5.2157600 & 6.0783030 & -3.4330080 \\
6.3972570 & 4.5010480 & -4.8721760 \\
6.8473470 & 2.5605530 & -4.0235280 \\
5.8144360 & 5.7553260 & -4.6586580 \\
4.7577550 & 7.0451380 & -3.2548880 \\
6.8541820 & 4.2743810 & -5.8304330 \\
5.8224930 & 6.4939430 & -5.4548050 \\
5.2649430 & -4.7027800 & -1.1836970 \\
3.8445870 & -5.8072570 & -2.6331440 \\
5.0999240 & -5.2306840 & -2.4488320 \\
3.5341350 & -6.3949470 & -3.8555600 \\
6.0783030 & -5.2157600 & -3.4330080 \\
4.5010480 & -6.3972570 & -4.8721760 \\
2.5605530 & -6.8473470 & -4.0235280 \\
5.7553260 & -5.8144360 & -4.6586580 \\
7.0451380 & -4.7577550 & -3.2548880 \\
4.2743810 & -6.8541820 & -5.8304330 \\
6.4939430 & -5.8224930 & -5.4548050 \\
-4.7027800 & -5.2649430 & -1.1836970 \\
-5.8072570 & -3.8445870 & -2.6331440 \\
-5.2306840 & -5.0999240 & -2.4488320 \\
-6.3949470 & -3.5341350 & -3.8555600 \\
-5.2157600 & -6.0783030 & -3.4330080 \\
-6.3972570 & -4.5010480 & -4.8721760 \\
-6.8473470 & -2.5605530 & -4.0235280 \\
-5.8144360 & -5.7553260 & -4.6586580 \\
-4.7577550 & -7.0451380 & -3.2548880 \\
-6.8541820 & -4.2743810 & -5.8304330 \\
-5.8224930 & -6.4939430 & -5.4548050 \\
-5.2649430 & 4.7027800 & -1.1836970 \\
-3.8445870 & 5.8072570 & -2.6331440 \\
-5.0999240 & 5.2306840 & -2.4488320 \\
-3.5341350 & 6.3949470 & -3.8555600 \\
-6.0783030 & 5.2157600 & -3.4330080 \\
-4.5010480 & 6.3972570 & -4.8721760 \\
-2.5605530 & 6.8473470 & -4.0235280 \\
-5.7553260 & 5.8144360 & -4.6586580 \\
-7.0451380 & 4.7577550 & -3.2548880 \\
-4.2743810 & 6.8541820 & -5.8304330 \\
-6.4939430 & 5.8224930 & -5.4548050 \\
& & \\
\hline
\end{tabular}

Benzofuran resorcinarene derivative in-endo (all-S,S, all- $R$ )-5 - DFT B3LYP/6-311+G(d,p)

Total Energy $=-3999.66681697$ a.u.

Cartesian coordinates

$\begin{array}{llll} & X & Z & \\ \text { C } & -1.2383980 & 3.0847860 & 1.4209640 \\ \text { C } & 0.0001160 & 2.5383390 & 1.7849200 \\ \text { H } & 0.0045030 & 1.7059400 & 2.4805140 \\ \text { C } & 1.2328730 & 3.0394700 & 1.3465100 \\ \text { C } & 1.2094290 & 4.1051720 & 0.4202250 \\ \text { C } & 0.0001160 & 4.7216830 & 0.0720710 \\ \text { C } & -1.1876730 & 4.2127000 & 0.5963810 \\ \text { O } & 2.3542570 & 4.6652250 & -0.1001670 \\ \text { H } & 2.9504950 & 3.9890180 & -0.4669060 \\ \text { O } & -2.3651230 & 4.9199800 & 0.3389110 \\ \text { C } & -2.5823670 & 5.3334130 & -0.9832520 \\ \text { H } & -3.4106690 & 6.0403030 & -0.9217730 \\ \text { C } & -1.3290620 & 5.8927910 & -1.7244700 \\ \text { H } & -1.5530350 & 6.9070490 & -2.0748410 \\ \text { C } & -0.0947700 & 5.9511130 & -0.7949390 \\ \text { H } & 0.8174720 & 6.0740760 & -1.3821760 \\ \text { C } & -2.5714170 & 2.5616530 & 1.9565150 \\ \text { H } & -3.3250350 & 3.2944400 & 1.6681670 \\ \text { C } & -2.5961150 & 2.4906310 & 3.5084530\end{array}$

$\begin{array}{llll}\mathrm{H} & -1.8292040 & 1.7886040 & 3.8586050 \\ \mathrm{H} & -3.5604890 & 2.0604690 & 3.8106510 \\ \mathrm{C} & -2.3995770 & 3.8331220 & 4.2429480 \\ \mathrm{H} & -1.4651490 & 4.2847170 & 3.8821570 \\ \mathrm{C} & -3.5426880 & 4.8256790 & 3.9734380 \\ \mathrm{H} & -3.6209410 & 5.0896300 & 2.9147080 \\ \mathrm{H} & -3.3875530 & 5.7551530 & 4.5332060 \\ \mathrm{H} & -4.5060660 & 4.4045330 & 4.2895660 \\ \mathrm{C} & -2.2482880 & 3.5854090 & 5.7528130 \\ \mathrm{H} & -3.1532470 & 3.1224340 & 6.1670540 \\ \mathrm{H} & -2.0794130 & 4.5239800 & 6.2927610 \\ \mathrm{H} & -1.4048860 & 2.9191070 & 5.9677790 \\ \mathrm{C} & 2.5616530 & 2.5714170 & 1.9565150 \\ \mathrm{C} & 3.0847860 & 1.2383980 & 1.4209640 \\ \mathrm{H} & 3.2944400 & 3.3250350 & 1.6681670 \\ \mathrm{C} & 2.4906310 & 2.5961150 & 3.5084530 \\ \mathrm{C} & 2.5383390 & -0.0001160 & 1.7849200 \\ \mathrm{H} & 1.7059400 & -0.0045030 & 2.4805140 \\ \mathrm{C} & 4.2127000 & 1.1876730 & 0.5963810 \\ \mathrm{H} & 1.7886040 & 1.8292040 & 3.8586050\end{array}$




\begin{tabular}{|c|c|c|}
\hline 2.0604690 & 3.5604890 & 3.8106510 \\
\hline 3.8331220 & 2.3995770 & 4.2429480 \\
\hline 4.2847170 & 1.4651490 & 3.8821570 \\
\hline 4.8256790 & 3.5426880 & 3.9734380 \\
\hline 5.0896300 & 3.6209410 & 2.9147080 \\
\hline 5.7551530 & 3.3875530 & 4.5332060 \\
\hline 4.4045330 & 4.5060660 & 4.2895660 \\
\hline 3.5854090 & 2.2482880 & 5.7528130 \\
\hline 3.1224340 & 3.1532470 & 6.1670540 \\
\hline 4.5239800 & 2.0794130 & 6.2927610 \\
\hline 2.9191070 & 1.4048860 & 5.9677790 \\
\hline 3.0394700 & -1.2328730 & 1.3465100 \\
\hline 4.1051720 & -1.2094290 & 0.4202250 \\
\hline 4.7216830 & -0.0001160 & 0.0720710 \\
\hline 4.6652250 & -2.3542570 & -0.1001670 \\
\hline 4.9199800 & 2.3651230 & 0.3389110 \\
\hline 5.3334130 & 2.5823670 & -0.9832520 \\
\hline 6.0403030 & 3.4106690 & -0.9217730 \\
\hline 5.8927910 & 1.3290620 & -1.7244700 \\
\hline 6.9070490 & 1.5530350 & -2.0748410 \\
\hline 5.9511130 & 0.0947700 & -0.7949390 \\
\hline 6.0740760 & -0.8174720 & -1.3821760 \\
\hline 1.2383980 & -3.0847860 & 1.4209640 \\
\hline-0.0001160 & -2.5383390 & 1.7849200 \\
\hline-0.0045030 & -1.7059400 & 2.4805140 \\
\hline 1.1876730 & -4.2127000 & 0.5963810 \\
\hline 3.9890180 & -2.9504950 & -0.4669060 \\
\hline 2.3651230 & -4.9199800 & 0.3389110 \\
\hline 2.5714170 & -2.5616530 & 1.9565150 \\
\hline 3.3250350 & -3.2944400 & 1.6681670 \\
\hline 2.5961150 & -2.4906310 & 3.5084530 \\
\hline 1.8292040 & -1.7886040 & 3.8586050 \\
\hline 3.5604890 & -2.0604690 & 3.8106510 \\
\hline 2.3995770 & -3.8331220 & 4.2429480 \\
\hline-1.2328730 & -3.0394700 & 1.3465100 \\
\hline-1.2094290 & -4.1051720 & 0.4202250 \\
\hline-0.0001160 & -4.7216830 & 0.0720710 \\
\hline-2.3542570 & -4.6652250 & -0.1001670 \\
\hline 2.5823670 & -5.3334130 & -0.9832520 \\
\hline 3.4106690 & -6.0403030 & -0.9217730 \\
\hline 1.3290620 & -5.8927910 & -1.7244700 \\
\hline 1.5530350 & -6.9070490 & -2.0748410 \\
\hline 0.0947700 & -5.9511130 & -0.7949390 \\
\hline-0.8174720 & -6.0740760 & -1.3821760 \\
\hline 1.4651490 & -4.2847170 & 3.8821570 \\
\hline-3.0847860 & -1.2383980 & 1.4209640 \\
\hline-2.5383390 & 0.0001160 & 1.7849200 \\
\hline-1.7059400 & 0.0045030 & 2.4805140 \\
\hline-4.2127000 & -1.1876730 & 0.5963810 \\
\hline-2.9504950 & -3.9890180 & -0.4669060 \\
\hline-4.9199800 & -2.3651230 & 0.3389110 \\
\hline-2.5616530 & -2.5714170 & 1.9565150 \\
\hline-3.2944400 & -3.3250350 & 1.6681670 \\
\hline-2.4906310 & -2.5961150 & 3.5084530 \\
\hline-1.7886040 & -1.8292040 & 3.8586050 \\
\hline-2.0604690 & -3.5604890 & 3.8106510 \\
\hline-3.8331220 & -2.3995770 & 4.2429480 \\
\hline-3.0394700 & 1.2328730 & 1.3465100 \\
\hline-4.1051720 & 1.2094290 & 0.4202250 \\
\hline-4.7216830 & 0.0001160 & 0.0720710 \\
\hline-4.6652250 & 2.3542570 & -0.1001670 \\
\hline-3.9890180 & 2.9504950 & -0.4669060 \\
\hline-4.2847170 & -1.4651490 & 3.8821570 \\
\hline-5.0896300 & -3.6209410 & 2.9147080 \\
\hline-4.8256790 & -3.5426880 & 3.9734380 \\
\hline-5.7551530 & -3.3875530 & 4.5332060 \\
\hline-4.4045330 & -4.5060660 & 4.2895660 \\
\hline
\end{tabular}

\begin{tabular}{|c|c|c|}
\hline-3.5854090 & -2.2482880 & 5.7528130 \\
\hline-3.1224340 & -3.1532470 & 6.1670540 \\
\hline-4.5239800 & -2.0794130 & 6.2927610 \\
\hline-2.9191070 & -1.4048860 & 5.9677790 \\
\hline-5.3334130 & -2.5823670 & -0.9832520 \\
\hline-6.0403030 & -3.4106690 & -0.9217730 \\
\hline-5.8927910 & -1.3290620 & -1.7244700 \\
\hline-6.9070490 & -1.5530350 & -2.0748410 \\
\hline-5.9511130 & -0.0947700 & -0.7949390 \\
\hline-6.0740760 & 0.8174720 & -1.3821760 \\
\hline 3.5426880 & -4.8256790 & 3.9734380 \\
\hline 3.6209410 & -5.0896300 & 2.9147080 \\
\hline 3.3875530 & -5.7551530 & 4.5332060 \\
\hline 4.5060660 & -4.4045330 & 4.2895660 \\
\hline 2.2482880 & -3.5854090 & 5.7528130 \\
\hline 3.1532470 & -3.1224340 & 6.1670540 \\
\hline 2.0794130 & -4.5239800 & 6.2927610 \\
\hline 1.4048860 & -2.9191070 & 5.9677790 \\
\hline 4.1918290 & 3.0576360 & -1.7505000 \\
\hline 4.9479580 & 1.2040720 & -2.8981040 \\
\hline 3.9975890 & 2.2205780 & -2.8289110 \\
\hline 4.9092210 & 0.3004490 & -3.9553460 \\
\hline 2.9809010 & 2.3687940 & -3.7632400 \\
\hline 3.9064990 & 0.4335350 & -4.9267280 \\
\hline 5.6465250 & -0.4946010 & -4.0319460 \\
\hline 2.9520970 & 1.4518160 & -4.8216350 \\
\hline 2.2399670 & 3.1547950 & -3.6722740 \\
\hline 3.8725230 & -0.2558030 & -5.7649780 \\
\hline 2.1725480 & 1.5383600 & -5.5726770 \\
\hline 3.0576360 & -4.1918290 & -1.7505000 \\
\hline 1.2040720 & -4.9479580 & -2.8981040 \\
\hline 2.2205780 & -3.9975890 & -2.8289110 \\
\hline 0.3004490 & -4.9092210 & -3.9553460 \\
\hline 2.3687940 & -2.9809010 & -3.7632400 \\
\hline 0.4335350 & -3.9064990 & -4.9267280 \\
\hline-0.4946010 & -5.6465250 & -4.0319460 \\
\hline 1.4518160 & -2.9520970 & -4.8216350 \\
\hline 3.1547950 & -2.2399670 & -3.6722740 \\
\hline-0.2558030 & -3.8725230 & -5.7649780 \\
\hline 1.5383600 & -2.1725480 & -5.5726770 \\
\hline-4.1918290 & -3.0576360 & -1.7505000 \\
\hline-4.9479580 & -1.2040720 & -2.8981040 \\
\hline-3.9975890 & -2.2205780 & -2.8289110 \\
\hline-4.9092210 & -0.3004490 & -3.9553460 \\
\hline-2.9809010 & -2.3687940 & -3.7632400 \\
\hline-3.9064990 & -0.4335350 & -4.9267280 \\
\hline-5.6465250 & 0.4946010 & -4.0319460 \\
\hline-2.9520970 & -1.4518160 & -4.8216350 \\
\hline-2.2399670 & -3.1547950 & -3.6722740 \\
\hline-3.8725230 & 0.2558030 & -5.7649780 \\
\hline-2.1725480 & -1.5383600 & -5.5726770 \\
\hline-3.0576360 & 4.1918290 & -1.7505000 \\
\hline-1.2040720 & 4.9479580 & -2.8981040 \\
\hline-2.2205780 & 3.9975890 & -2.8289110 \\
\hline-0.3004490 & 4.9092210 & -3.9553460 \\
\hline-2.3687940 & 2.9809010 & -3.7632400 \\
\hline-0.4335350 & 3.9064990 & -4.9267280 \\
\hline 0.4946010 & 5.6465250 & -4.0319460 \\
\hline-1.4518160 & 2.9520970 & -4.8216350 \\
\hline-3.1547950 & 2.2399670 & -3.6722740 \\
\hline 0.2558030 & 3.8725230 & -5.7649780 \\
\hline-1.5383600 & 2.1725480 & -5.5726770 \\
\hline-0.1812940 & 6.8406350 & -0.1552180 \\
\hline-6.8406350 & -0.1812940 & -0.1552180 \\
\hline 0.1812940 & -6.8406350 & -0.1552180 \\
\hline 6.8406350 & 0.1812940 & -0.1552180 \\
\hline
\end{tabular}

Benzofuran resorcinarene derivative in-exo (all- $R, R$, all- $R$ )-5 - DFT B3LYP/6-311+G(d,p)

Total Energy = -3999.66174688 a.u.

Cartesian coordinates

$\begin{array}{llll} & X & Y & Z \\ C & -2.0854150 & 2.5414450 & 1.2988140 \\ C & -0.7777480 & 2.4891180 & 1.7884930 \\ H & -0.5744770 & 1.8309960 & 2.6260700 \\ C & 0.2779520 & 3.2520520 & 1.2760930 \\ C & -0.0082850 & 4.0907160 & 0.1832640 \\ C & -1.3092610 & 4.1983900 & -0.3391420 \\ C & -2.3167310 & 3.4207740 & 0.2287830\end{array}$

$\begin{array}{llll}\mathrm{O} & 0.9385220 & 4.8780430 & -0.4158890 \\ \mathrm{H} & 1.8315980 & 4.5360480 & -0.2271450 \\ \mathrm{O} & -3.6265290 & 3.4610520 & -0.2619000 \\ \mathrm{C} & -3.9633210 & 4.1647060 & -1.4267280 \\ \mathrm{C} & -2.8165450 & 4.6954540 & -2.3053590 \\ \mathrm{H} & -2.4993460 & 3.9194630 & -3.0088460 \\ \mathrm{C} & -1.6219830 & 5.1657960 & -1.4507280 \\ \mathrm{H} & -0.7438920 & 5.3094560 & -2.0843720\end{array}$




\begin{tabular}{|c|c|c|}
\hline-3.2109330 & 1.6844100 & 1.8897760 \\
\hline-4.1520090 & 2.1686550 & 1.6204120 \\
\hline-3.2061070 & 1.6472220 & 3.4387110 \\
\hline-2.2708290 & 1.2096980 & 3.8071730 \\
\hline-4.0032010 & 0.9613860 & 3.7559400 \\
\hline-3.4130880 & 3.0070460 & 4.1383670 \\
\hline-2.6365250 & 3.6964560 & 3.7801870 \\
\hline-4.7820520 & 3.6332890 & 3.8246170 \\
\hline-4.9033310 & 3.8611330 & 2.7611360 \\
\hline-4.9113150 & 4.5727660 & 4.3733410 \\
\hline-5.5970990 & 2.9603220 & 4.1214970 \\
\hline-3.2327120 & 2.8444800 & 5.6565380 \\
\hline-3.9867500 & 2.1632720 & 6.0714390 \\
\hline-3.3354780 & 3.8059240 & 6.1718540 \\
\hline-2.2449160 & 2.4379360 & 5.9027790 \\
\hline 1.6844100 & 3.2109330 & 1.8897760 \\
\hline 2.5414450 & 2.0854150 & 1.2988140 \\
\hline 2.1686550 & 4.1520090 & 1.6204120 \\
\hline 1.6472220 & 3.2061070 & 3.4387110 \\
\hline 2.4891180 & 0.7777480 & 1.7884930 \\
\hline 1.8309960 & 0.5744770 & 2.6260700 \\
\hline 3.4207740 & 2.3167310 & 0.2287830 \\
\hline 1.2096980 & 2.2708290 & 3.8071730 \\
\hline 0.9613860 & 4.0032010 & 3.7559400 \\
\hline 3.0070460 & 3.4130880 & 4.1383670 \\
\hline 3.6964560 & 2.6365250 & 3.7801870 \\
\hline 3.6332890 & 4.7820520 & 3.8246170 \\
\hline 3.8611330 & 4.9033310 & 2.7611360 \\
\hline 4.5727660 & 4.9113150 & 4.3733410 \\
\hline 2.9603220 & 5.5970990 & 4.1214970 \\
\hline 2.8444800 & 3.2327120 & 5.6565380 \\
\hline 2.1632720 & 3.9867500 & 6.0714390 \\
\hline 3.8059240 & 3.3354780 & 6.1718540 \\
\hline 2.4379360 & 2.2449160 & 5.9027790 \\
\hline 3.2520520 & -0.2779520 & 1.2760930 \\
\hline 4.0907160 & 0.0082850 & 0.1832640 \\
\hline 4.1983900 & 1.3092610 & -0.3391420 \\
\hline 4.8780430 & -0.9385220 & -0.4158890 \\
\hline 3.4610520 & 3.6265290 & -0.2619000 \\
\hline 4.1647060 & 3.9633210 & -1.4267280 \\
\hline 4.6954540 & 2.8165450 & -2.3053590 \\
\hline 3.9194630 & 2.4993460 & -3.0088460 \\
\hline 5.1657960 & 1.6219830 & -1.4507280 \\
\hline 5.3094560 & 0.7438920 & -2.0843720 \\
\hline 2.0854150 & -2.5414450 & 1.2988140 \\
\hline 0.7777480 & -2.4891180 & 1.7884930 \\
\hline 0.5744770 & -1.8309960 & 2.6260700 \\
\hline 2.3167310 & -3.4207740 & 0.2287830 \\
\hline 4.5360480 & -1.8315980 & -0.2271450 \\
\hline 3.6265290 & -3.4610520 & -0.2619000 \\
\hline 3.2109330 & -1.6844100 & 1.8897760 \\
\hline 4.1520090 & -2.1686550 & 1.6204120 \\
\hline 3.2061070 & -1.6472220 & 3.4387110 \\
\hline 2.2708290 & -1.2096980 & 3.8071730 \\
\hline 4.0032010 & -0.9613860 & 3.7559400 \\
\hline 3.4130880 & -3.0070460 & 4.1383670 \\
\hline-0.2779520 & -3.2520520 & 1.2760930 \\
\hline 0.0082850 & -4.0907160 & 0.1832640 \\
\hline 1.3092610 & -4.1983900 & -0.3391420 \\
\hline-0.9385220 & -4.8780430 & -0.4158890 \\
\hline 3.9633210 & -4.1647060 & -1.4267280 \\
\hline 2.8165450 & -4.6954540 & -2.3053590 \\
\hline 2.4993460 & -3.9194630 & -3.0088460 \\
\hline 1.6219830 & -5.1657960 & -1.4507280 \\
\hline 0.7438920 & -5.3094560 & -2.0843720 \\
\hline 2.6365250 & -3.6964560 & 3.7801870 \\
\hline-2.5414450 & -2.0854150 & 1.2988140 \\
\hline-2.4891180 & -0.7777480 & 1.7884930 \\
\hline-1.8309960 & -0.5744770 & 2.6260700 \\
\hline-3.4207740 & -2.3167310 & 0.2287830 \\
\hline-1.8315980 & -4.5360480 & -0.2271450 \\
\hline-3.4610520 & -3.6265290 & -0.2619000 \\
\hline-1.6844100 & -3.2109330 & 1.8897760 \\
\hline-2.1686550 & -4.1520090 & 1.6204120 \\
\hline-1.6472220 & -3.2061070 & 3.4387110 \\
\hline-1.2096980 & -2.2708290 & 3.8071730 \\
\hline-0.9613860 & -4.0032010 & 3.7559400 \\
\hline-3.0070460 & -3.4130880 & 4.1383670 \\
\hline-3.2520520 & 0.2779520 & 1.2760930 \\
\hline
\end{tabular}

\begin{tabular}{|c|c|c|}
\hline-4.0907160 & -0.0082850 & 0.1832640 \\
\hline-4.1983900 & -1.3092610 & -0.3391420 \\
\hline-4.8780430 & 0.9385220 & -0.4158890 \\
\hline-4.5360480 & 1.8315980 & -0.2271450 \\
\hline-3.6964560 & -2.6365250 & 3.7801870 \\
\hline-3.8611330 & -4.9033310 & 2.7611360 \\
\hline-3.6332890 & -4.7820520 & 3.8246170 \\
\hline-4.5727660 & -4.9113150 & 4.3733410 \\
\hline-2.9603220 & -5.5970990 & 4.1214970 \\
\hline-2.8444800 & -3.2327120 & 5.6565380 \\
\hline-2.1632720 & -3.9867500 & 6.0714390 \\
\hline-3.8059240 & -3.3354780 & 6.1718540 \\
\hline-2.4379360 & -2.2449160 & 5.9027790 \\
\hline-4.1647060 & -3.9633210 & -1.4267280 \\
\hline-4.6954540 & -2.8165450 & -2.3053590 \\
\hline-3.9194630 & -2.4993460 & -3.0088460 \\
\hline-5.1657960 & -1.6219830 & -1.4507280 \\
\hline-5.3094560 & -0.7438920 & -2.0843720 \\
\hline 4.7820520 & -3.6332890 & 3.8246170 \\
\hline 4.9033310 & -3.8611330 & 2.7611360 \\
\hline 4.9113150 & -4.5727660 & 4.3733410 \\
\hline 5.5970990 & -2.9603220 & 4.1214970 \\
\hline 3.2327120 & -2.8444800 & 5.6565380 \\
\hline 3.9867500 & -2.1632720 & 6.0714390 \\
\hline 3.3354780 & -3.8059240 & 6.1718540 \\
\hline 2.2449160 & -2.4379360 & 5.9027790 \\
\hline 5.3289020 & 4.7495160 & -1.0673460 \\
\hline 5.8608040 & 3.5325600 & -2.9552060 \\
\hline 6.1933700 & 4.6146010 & -2.1310390 \\
\hline 6.6355010 & 3.2520520 & -4.0744290 \\
\hline 7.2821730 & 5.4387500 & -2.3823070 \\
\hline 7.7390140 & 4.0729560 & -4.3551810 \\
\hline 6.3953430 & 2.4129910 & -4.7219430 \\
\hline 8.0565110 & 5.1453030 & -3.5142310 \\
\hline 7.5150190 & 6.2730060 & -1.7294040 \\
\hline 8.3499420 & 3.8746040 & -5.2302800 \\
\hline 8.9144160 & 5.7705070 & -3.7439180 \\
\hline 4.7495160 & -5.3289020 & -1.0673460 \\
\hline 3.5325600 & -5.8608040 & -2.9552060 \\
\hline 4.6146010 & -6.1933700 & -2.1310390 \\
\hline 3.2520520 & -6.6355010 & -4.0744290 \\
\hline 5.4387500 & -7.2821730 & -2.3823070 \\
\hline 4.0729560 & -7.7390140 & -4.3551810 \\
\hline 2.4129910 & -6.3953430 & -4.7219430 \\
\hline 5.1453030 & -8.0565110 & -3.5142310 \\
\hline 6.2730060 & -7.5150190 & -1.7294040 \\
\hline 3.8746040 & -8.3499420 & -5.2302800 \\
\hline 5.7705070 & -8.9144160 & -3.7439180 \\
\hline-5.3289020 & -4.7495160 & -1.0673460 \\
\hline-5.8608040 & -3.5325600 & -2.9552060 \\
\hline-6.1933700 & -4.6146010 & -2.1310390 \\
\hline-6.6355010 & -3.2520520 & -4.0744290 \\
\hline-7.2821730 & -5.4387500 & -2.3823070 \\
\hline-7.7390140 & -4.0729560 & -4.3551810 \\
\hline-6.3953430 & -2.4129910 & -4.7219430 \\
\hline-8.0565110 & -5.1453030 & -3.5142310 \\
\hline-7.5150190 & -6.2730060 & -1.7294040 \\
\hline-8.3499420 & -3.8746040 & -5.2302800 \\
\hline-8.9144160 & -5.7705070 & -3.7439180 \\
\hline-4.7495160 & 5.3289020 & -1.0673460 \\
\hline-3.5325600 & 5.8608040 & -2.9552060 \\
\hline-4.6146010 & 6.1933700 & -2.1310390 \\
\hline-3.2520520 & 6.6355010 & -4.0744290 \\
\hline-5.4387500 & 7.2821730 & -2.3823070 \\
\hline-4.0729560 & 7.7390140 & -4.3551810 \\
\hline-2.4129910 & 6.3953430 & -4.7219430 \\
\hline-5.1453030 & 8.0565110 & -3.5142310 \\
\hline-6.2730060 & 7.5150190 & -1.7294040 \\
\hline-3.8746040 & 8.3499420 & -5.2302800 \\
\hline-5.7705070 & 8.9144160 & -3.7439180 \\
\hline 4.6251380 & -3.5003530 & -1.9964860 \\
\hline 3.5003530 & 4.6251380 & -1.9964860 \\
\hline-3.5003530 & -4.6251380 & -1.9964860 \\
\hline-4.6251380 & 3.5003530 & -1.9964860 \\
\hline-6.1502210 & -1.8540270 & -1.0230040 \\
\hline 1.8540270 & -6.1502210 & -1.0230040 \\
\hline 6.1502210 & 1.8540270 & -1.0230040 \\
\hline & & \\
\hline
\end{tabular}


${ }^{1}$ Frisch, M. J.; Trucks, G. W.; Schlegel, H. B.; Scuseria, G. E.; Robb, M. A.; Cheeseman, J. R.; Scalmani, G.; Barone, V.; Mennucci, B.; Petersson, G. A.; Nakatsuji, H.; Caricato, M.; Li, X.; Hratchian, H. P.; Izmaylov, A. F.; Bloino, J.; Zheng, G.; Sonnenberg, J. L.; Hada, M.; Ehara, M.; Toyota, K.; Fukuda, R.; Hasegawa, J.; Ishida, M.; Nakajima, T.; Honda, Y.; Kitao, O.; Nakai, H.; Vreven, T.; Montgomery, J. A., Jr.; Peralta, J. E.; Ogliaro, F.; Bearpark, M.; Heyd, J. J.; Brothers, E.; Kudin, K. N.; Staroverov, V. N.; Kobayashi, R.; Normand, J.; Raghavachari, K.; Rendell, A.; Burant, J. C.; Iyengar, S. S.; Tomasi, J.; Cossi, M.; Rega, N.; Millam, J. M.; Klene, M.; Knox, J. E.; Cross, J. B.; Bakken, V.; Adamo, C.; Jaramillo, J.; Gomperts, R.; Stratmann, R. E.; Yazyev, O.; Austin, A. J.; Cammi, R.; Pomelli, C.; Ochterski, J. W.; Martin, R. L.; Morokuma, K.; Zakrzewski, V. G.; Voth, G. A.; Salvador, P.; Dannenberg, J. J.; Dapprich, S.; Daniels, A. D.; Farkas, Ö.; Foresman, J. B.; Ortiz, J. V.; Cioslowski, J.; Fox, D. J. Gaussian 09, Revision D.01, Gaussian, Inc., Wallingford CT, 2009. 